Authorship note: $][$ and C shared senior authorship.

Conflict of interest: CDW, AR, and JC are inventors on a patent application (no. W02019070407) for eicosanoids as biomarkers of senescence. AV is currently an employee at UNITY Biotechnology. HAC is the scientific founder of Pliant Therapeutics, which develops antifibrotic drugs and owns equity in the company. $J C$ is the scientific founder of UNITY Biotechnology, which develops senolytic and other drugs to combat aging, owns equity in the company, and receives research funding from the company. JC is also named on several planned, pending, and awarded patents on the use of small molecules to eradicate senescent cells CJLS has served as a consultant for UNITY Biotechnology and Gordian.

Copyright: (c) 2019, American Society for Clinical Investigation.

Submitted: May 8, 2019

Accepted: October 29, 2019

Published: December 19, 2019

Reference information: JCl Insight. 2019;4(24):e130056. https://doi.org/10.1172/jci. insight.130056.

\section{Secretion of leukotrienes by senescent lung fibroblasts promotes pulmonary fibrosis}

\author{
Christopher D. Wiley, ${ }^{1}$ Alexis N. Brumwell, ${ }^{2}$ Sonnet S. Davis, ${ }^{1}$ Julia R. Jackson, ${ }^{2}$ Alexis Valdovinos, ${ }^{1}$ \\ Cheresa Calhoun, ${ }^{3}$ Fatouma Alimirah, ${ }^{1}$ Carlos A. Castellanos, ${ }^{2}$ Richard Ruan, ${ }^{2}$ Ying Wei, ${ }^{2}$ \\ Harold A. Chapman, ${ }^{2}$ Arvind Ramanathan, ${ }^{1,4}$ Judith Campisi, ${ }^{1,5}$ and Claude Jourdan Le Saux ${ }^{2,3}$ \\ ${ }^{1}$ Buck Institute for Research on Aging, Novato, California, USA. ${ }^{2}$ UCSF, San Francisco, California, USA. ${ }^{3}$ University of \\ Texas Health Science Center at San Antonio, San Antonio, Texas, USA. ${ }^{4}$ Institute for Stem Cell Biology and Regenerative \\ Medicine (inStem), Rajiv Gandhi Nagar, Kodigehalli, Bengaluru, Karnataka, India. ${ }^{5}$ Lawrence Berkeley National Laboratory, \\ Berkeley, California, USA.
}

\begin{abstract}
Accumulation of senescent cells is associated with the progression of pulmonary fibrosis, but mechanisms accounting for this linkage are not well understood. To explore this issue, we investigated whether a class of biologically active profibrotic lipids, the leukotrienes (LT), is part of the senescence-associated secretory phenotype. The analysis of conditioned medium (CM), lipid extracts, and gene expression of LT biosynthesis enzymes revealed that senescent cells secreted LT, regardless of the origin of the cells or the modality of senescence induction. The synthesis of LT was biphasic and followed by antifibrotic prostaglandin (PC) secretion. The LT-rich CM of senescent lung fibroblasts (IMR-90) induced profibrotic signaling in naive fibroblasts, which were abrogated by inhibitors of ALOX5, the principal enzyme in LT biosynthesis. The bleomycin-induced expression of genes encoding LT and PG synthases, level of cysteinyl LT in the bronchoalveolar lavage, and overall fibrosis were reduced upon senescent cell removal either in a genetic mouse model or after senolytic treatment. Quantification of $\mathrm{ALOX5}^{+}$cells in lung explants obtained from idiopathic pulmonary fibrosis (IPF) patients indicated that half of these cells were also senescent (p16 $6^{\operatorname{lnk} 4 a+}$ ). Unlike human fibroblasts from unused donor lungs made senescent by irradiation, senescent IPF fibroblasts secreted LTs but failed to synthesize PGs. This study demonstrates for the first time to our knowledge that senescent cells secrete functional LTs, significantly contributing to the LT pool known to cause or exacerbate IPF.
\end{abstract}

\section{Introduction}

The development of fibrosis is a frequent complication of a number of chronic lung diseases, including idiopathic pulmonary fibrosis (IPF) and contributes to their morbidity and mortality. Characterized by an irreversible destruction of their lung architecture associated with a progressive accumulation of fibrotic tissue, the median survival for IPF patients is 3-5 years from diagnosis (1). Indeed, no effective therapies are available to stop the progression of fibrotic tissue remodeling, despite 2 US Food and Drug Administration-approved (FDA-approved) therapeutic options (1). The underlying pathobiology is thought to be due to repetitive injuries and the inability of the epithelium to repair. Although once not considered critical to IPF initiation or progression, inflammation - especially macrophage accumulation — has reemerged as a potentially important contributor to fibrosis progression $(2,3)$. A recent study showed that severe innate and adaptive inflammatory infiltrates were detected in IPF patients and were predictive of the disease progression (4). In addition to mediators derived from activated epithelial and immune cells, recent data implicate senescent and necroptotic cells as sources of those proinflammatory mediators (5).

For some time, senescence has been thought to contribute to the pathology of IPF without strong supportive data. However, accumulating evidence links the extent of senescent cells in fibrotic tissue with the severity of IPF (5). Independent studies show that markers of cellular senescence (p53, p21 ${ }^{\mathrm{WAF}}$, and p16 ${ }^{\text {Ink4a }}$ ) are upregulated in type II alveolar epithelial cells (AECIIs) in IPF lungs within the remodeled fibrotic areas (5-7). The presence of relatively short telomeres, which can lead to cellular senescence, was 
reported in AECIIs and fibroblasts in IPF lungs $(8,9)$. Recent findings indicate that senescent fibroblasts secrete PDGF receptor ligands, supporting their role in fibrosis (10). Furthermore, limiting the number of these senescent cells is beneficial in controlling the extent of remodeling in experimental models of lung injury (11-15). However, the mechanisms by which senescent cells contribute to the pathobiology of IPF remain incompletely understood. Many deleterious effects of senescent cells result from the secretion of myriad biologically active proteins, including inflammatory cytokines and chemokines, matrix metalloproteinases, and growth factors $(16,17)$. This senescence-associated secretory phenotype (SASP) has been characterized primarily in the context of secreted proteins. However, the expression of 5-lipoxygenase (ALOX5) and cyclooxygenase 2 (COX2) are reported in senescent fibroblasts, raising the possibility that biologically active signaling lipids could be derived from senescent cells $(18,19)$. Eicosanoids are divided in several families, of which the most relevant for fibrosis are the leukotrienes (LTs) and prostaglandins (PGs). COX2, the rate limiting enzyme in PG biosynthesis, is highly upregulated during both normal and stress-induced fibroblast senescence (18). However, secretion of the antifibrotic PGs has been found to be low in fibrotic models and IPF (20-23). Indeed, IPF-derived cultured fibroblasts failed to increase COX2 protein expression or COX activity in response to stimuli, in contrast to normal lung fibroblasts (24). Additionally, the LTs, a second class of biologically active signaling lipids, can modulate diverse physiological responses, including inflammation and fibrosis (25-27). To expand our knowledge of the SASP and its relevance to fibrosis, here we investigate LT and PG secretion by senescent cells.

\section{Results}

Senescent fibroblasts secrete LTs as part of their SASP. We first investigated whether a class of biologically active signaling profibrotic lipids, namely the LT family, are part of the SASP. We interrogated the status of cytosolic phospholipase A2 (cPLA2), the major enzyme that releases arachidonic acid from the plasma membrane (28). cPLA2 is activated by phosphorylation on serine 505 by p38MAPK (29), which is activated in senescent cells (30). As expected, irradiated lung fibroblasts confirmed to be senescent by p21 ${ }^{\text {WAF1 }}$ expression (Figure 1A) showed activation (phosphorylation) of p38MAPK and cPLA2 (Figure 1B). Because p38MAPK signaling often culminates in increased transcription, we used quantitative PCR (qPCR) to measure the abundance of mRNAs encoding genes that specifically participate in eicosanoid synthesis. Compared with quiescent cells, senescent cells expressed higher levels of several of these genes (Figure 1C), including those encoding LT synthases, such as ALOX5 (5-LO), ALOX12, ALOX15, ALOX$5 A P, L T C 4 S, L T A 4 H$, and $A L O X 15 B$ (Figure 1C). To confirm the activation of the biosynthesis of LT in the senescent fibroblasts, increased levels of ALOX5 synthesis and activation as showed by a significant increased phosphorylated ALOX5 (6.5-fold increased) were measured in senescent cells compared with quiescent cells (Figure 1D). In addition, a significant 3-fold increase of cysteinyl LT level was measured in conditioned medium (CM) from senescent fibroblasts and significantly inhibited in the presence of the ALOX5 inhibitors BW-B70C (BW) or zileuton (Zil) (Figure 1E). Additionally, in agreement with our RNA data, lipid extracts from senescent cells showed elevated levels of LTB4 (Figure 1F). A time course revealed the complex dynamics that govern the expression of eicosanoid synthesis genes during the senescence response, as exemplified by the time course expression of LT synthases (ALOX5 and LTA4H) and PG synthases (COX2 or PTGS2 and PTGES). The LT expression was biphasic, showing a large increase 2 days after irradiation, followed by a decline and a smaller peak of expression 10-20 days following irradiation (Figure 1G). Interestingly, in the late phase of senescence (between 15-20 days following irradiation), PG biosynthesis enzymes, including COX2 (PTGS2), were increased (Figure 1H).

LT expression is a widespread component of the SASP. To determine whether the cell origin or the mode of senescence induction affected the LT biosynthesis activation, we also measured cysteinyl LT levels in human umbilical vein cells (HUVEC) and human liver carcinoma cells (HEPG2). We cultured the cells under standard conditions specific to each cell lines. A 3-fold and 2-fold increase in LT expression was measured in irradiated HUVEC and HEPG2 cell lines, respectively (Figure 2A).

We induced senescence in IMR-90 fibroblasts following different modalities: mitochondria dysfunctional associated senescence (31), exposing them to a relatively high dose (10 Gy) of ionizing radiation ( $\gamma$ RA [ $\gamma$-irradiation]), oncogene induced (Ras), and oxidative stress (bleomycin). Each of these modalities was able to promote the secretion of LTs either evaluated by the measurement of cysteinyl LT in the CM (Figure 2B) or the activation of the expression of enzymes involved in the biosynthesis of LT (Figure 2C). Interestingly, these senescent cells also expressed enzymes related to the PG pathway (Figure 2D). Bleo- 

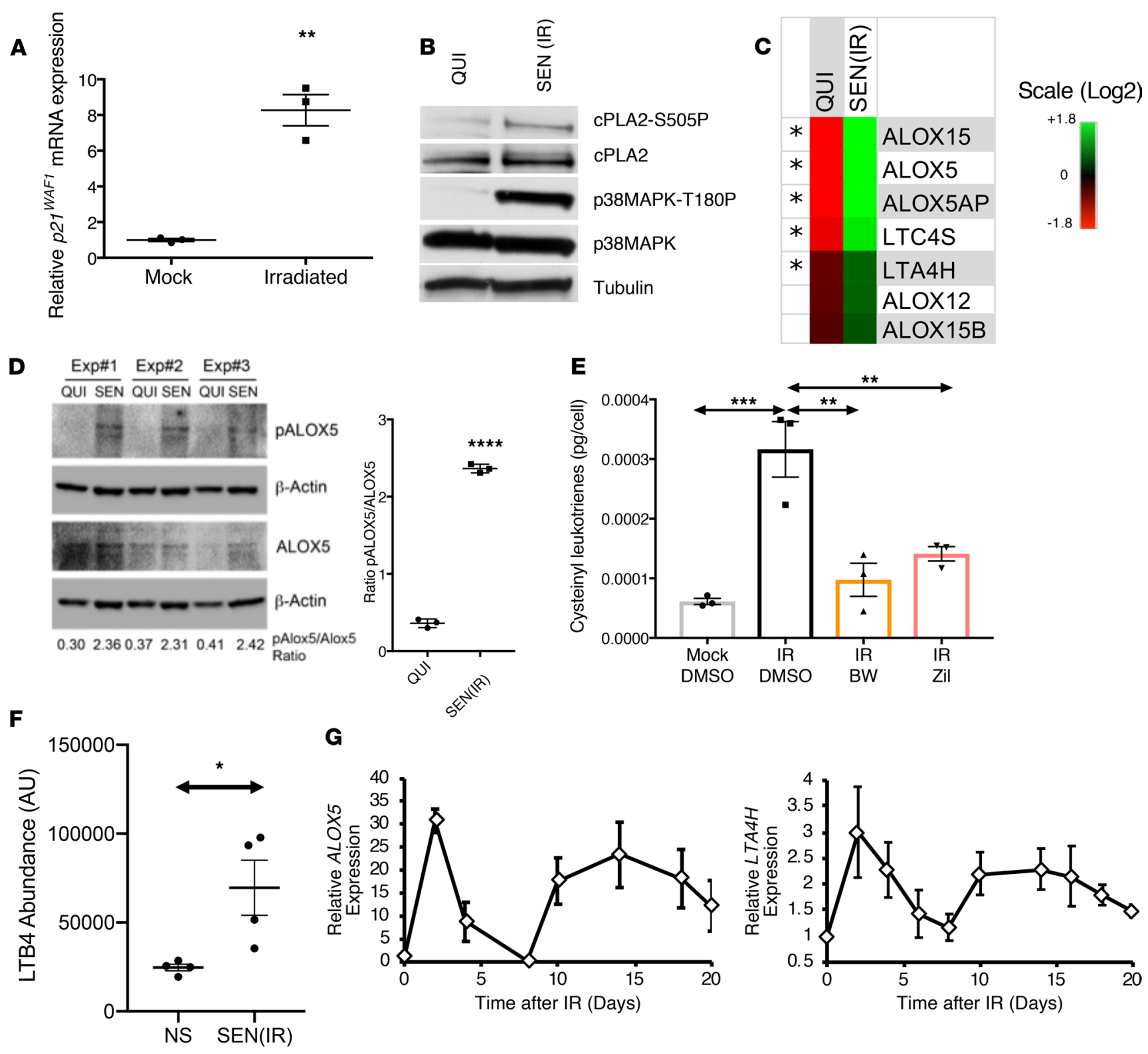

G
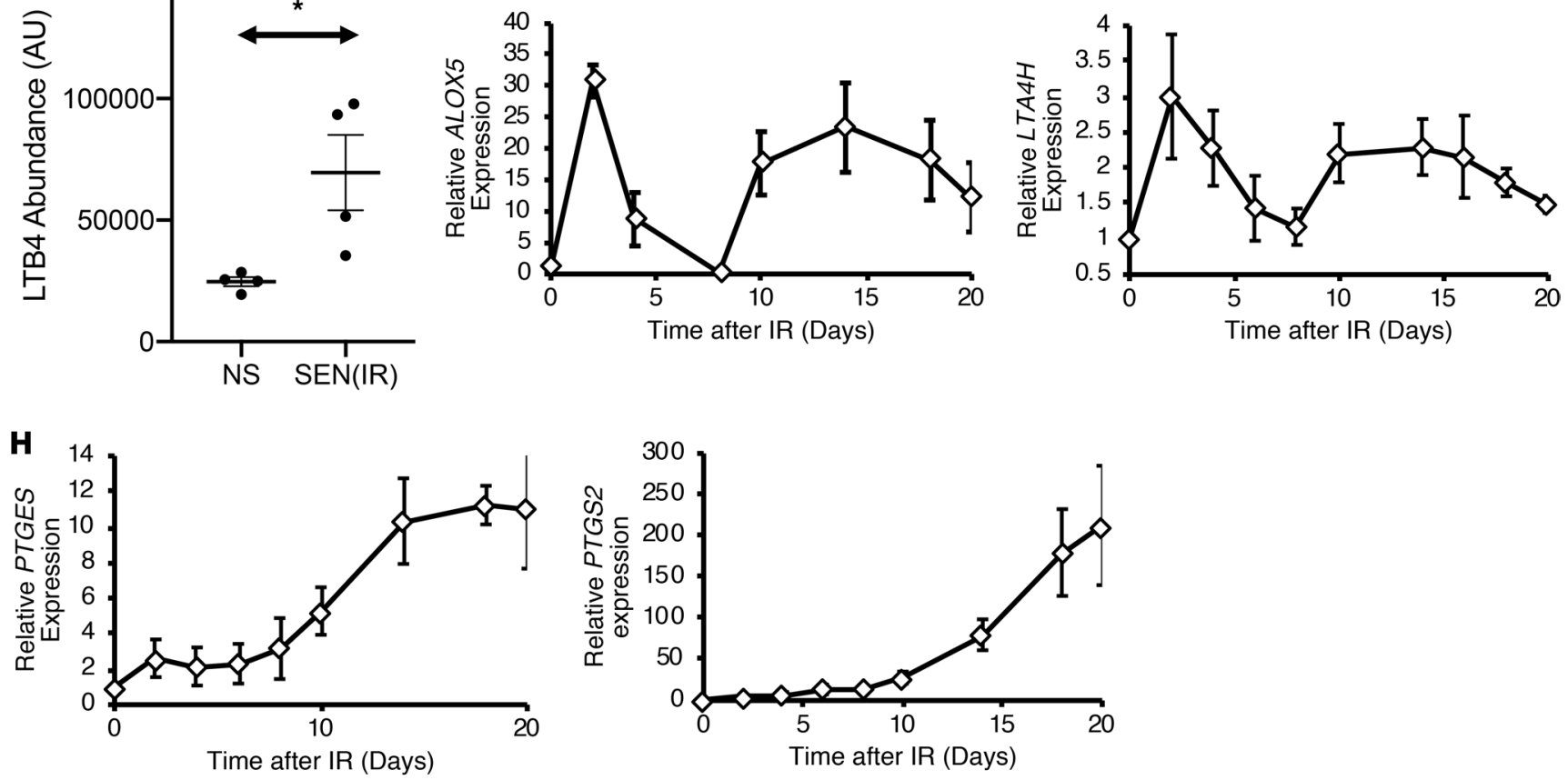
Figure 1. Senescent cells synthesize eicosanoids in a time-dependent manner. Senescence was induced in human lung fibroblasts (IMR-90) using irradiation (10 Gy). Total RNA was isolated from mock irradiated, quiescent (QUI, cell cultured in $0.2 \%$ serum/DMEM), and irradiated cells after 10 days of culture, reverse transcribed, and analyzed by quantitative PCR. Signal was normalized to tubulin mRNA. (A) Increased p27 ${ }^{\text {WAF1 }}$ mRNA level confirmed the induction of senescence in irradiated cells compared with mock irradiated ones. (B) Proteins were extracted from QUI and irradiated senescent (SEN[IR]) cells and analyzed by Western blot for CPLA2 (phosphorylated on serine 505 or total cPLA2), p38MAPK (phosphorylated on threonine 180 or total), and tubulin (control). (C) Panel of expression of genes encoding leukotriene synthesis enzymes. (D) Lysates from QUI and 10-days postirradiation senescent IMR-90 fibroblasts were blotted for ALOX5 (total and phosphorylated on serine 271). Quantification of Western blot bands were first normalized to $\beta$-actin and activation of ALOX5 is reported as the ratio p-ALOX5/ALOX5. (E) After ionizing radiation, fibroblasts were treated with DMSO (vehicle) or the ALOX5 inhibitors zileuton (Zil, $50 \mu \mathrm{M}$ ) or BW-B70C (BW, $10 \mu \mathrm{M}$ ) for 10 consecutive days, and conditioned medium (CM) was collected. Levels of cysteinyl leukotriene secreted in CM was measured by ELISA. (F) Intracellular level of leukotriene B4 measured by ELISA. (G) Time course expression of ALOX5 and LTA4H mRNA. (H) Time course expression of PTCS2 or COX2 and PTCES mRNA. Data are presented as mean \pm SEM of at least 3 replicates. Statistical analyses were performed using Student's $t$ test (A, C, and $\mathbf{D})$, 1-way ANOVA (D), or individual 2-tailed unpaired Student's $t$ test $(\mathbf{E}$ and $\mathbf{F}){ }^{*} P \leq 0.05$; ${ }^{* *} P \leq 0.01$; ${ }^{* *} P$ $\leq 0.001 ;{ }^{* * *} P \leq 0.0001$.

mycin-induced senescence was confirmed by the increased expression of $p 16^{\text {thk4a }}$ and $p 21^{\text {Wafl }} \mathrm{mRNA}$ (Figure 2E). Thus, the secretion of LT appears to be a feature of the SASP, independently of the cell type or the modality of senescence induction.

Senescent fibroblasts promote fibrosis by secreting LTs as part of their SASP. Since the enzymatic machinery leading to the biosynthesis of LTs and PGs is activated and cysteinyl LT is released in senescent fibroblasts, we sought to test whether the secreted LT and PGs had a functional effect on naive fibroblasts. We generated CM from control (day 0) or senescent cells, 2 days (2-day CM) or 20 days (20-day CM) after irradiation, to determine whether the secreted LTs and PGs from senescent cells could alter fibrotic responses. Prior to collecting the CM, the senescent cells were treated with NS-398 to inhibit PG biosynthesis or Zil to prevent LT biosynthesis. Naive nonsenescent IMR-90 fibroblasts were then treated with the CMs, and the activation of COL1A2 and $\alpha S M A$ gene expression was assessed as surrogates for the evaluation of fibrotic response (Figure $3 \mathrm{~A}$ ). The upregulation of $C O L 1 A 2 \mathrm{mRNA}$ level measured after treatment with 2-day $\mathrm{CM}$ was prevented with 2-day $\mathrm{CM}$ from the senescent cells treated with Zil (2-day + Zil CM). Further, upregulation of COL1A2 mRNA was lower using 20-day CM, consistent with declined expression of LT in the 20-day postirradiation SASP. Treatment of the naive fibroblasts with 2-day or 20-day CM + NS-398 (2-day + NS-398 or 20-day + NS-398 CM) did not affect the upregulation of COL1A2 mRNA expression (Figure 3B). Similarly, the expression of $\alpha$-smooth muscle actin ( $\alpha \mathrm{SMA}$, Acta2), a marker of myofibroblast differentiation, was upregulated in the presence of the 2-day CM and significantly reduced after treatment with 20-day CM. The downregulation of aSMA mRNA expression was abrogated if the naive fibroblasts were treated with 20-day + NS-398 CM, suggesting that the decline of $\alpha S M A$ expression was, in part, controlled by the released of PGs by the senescent cells (Figure 3C). However, $\alpha S M A$ mRNA expression was not affected by $\mathrm{CMs}+\mathrm{Zil}$, suggesting that the upregulation of $\alpha S M A$ mRNA was independent of LT expression. Of note, TGF- $\beta$ mRNA levels were not upregulated in irradiated senescence cells, and treatment with LT inhibitors did not change its expression (see below). It is, therefore, unlikely that the profibrotic effects are related to the presence of TGF- $\beta$ in the SASP.

Since TGF- $\beta$ is the master regulator of fibrosis, we also treated the naive fibroblasts with TGF- $\beta$ and the CMs. The level of COL1A2 mRNA expression was comparable when the naive fibroblasts were costimulated with 2-day CM or with 2-day CM + TGF- $\beta$, and inhibition of LT synthesis by Zil prevented the collagen expression induction using 2-day CM + TGF- $\beta$ + Zil (Supplemental Figure 1B; supplemental material available online with this article; https://doi.org/10.1172/jci.insight.130056DS1). The presence of TGF- $\beta$ was required to maintain collagen expression when treated with 20-day CM (Supplemental Figure 1B). Treatment with TGF- $\beta$ alone did not affect $\alpha S M A$ mRNA expression. Twenty-day CM was less effective at inducing $\alpha S M A$ mRNA expression than 2-day $\mathrm{CM}$, but treating senescent cells with 20-day $\mathrm{CM}+2$-day CM + TGF- $\beta$ + NS-398 prevented the downregulation of $\alpha S M A$ mRNA expression (Supplemental Figure $1 C)$, consistent again with findings that PGs antagonize myofibroblast activation $(32,33)$.

Because LTs trigger cellular signaling through membrane-bound GPCRs, we then profiled known LT receptors in naive quiescent fibroblasts to determine which type was the most likely to trigger the profibrotic responses in naive fibroblasts. We first detected the mRNA expression of several LT receptors (Supplemental Figure 1A). Among all the ones we tested, the LT B4 receptors B1 and B2 (BLT1, BLT2), the GPCRs 99 and 17 (GPR99, GPR17), the adenosine diphosphate-reactive (ADP-reactive) purinergic (P2Y ${ }_{12}$ ) receptor, and the cysteinyl LT receptors 1 and 2 (CYSLTR1, CYSLTR2) were expressed. This suggests that the naive fibroblasts were capable of responding to multiple members of the LT family. To investigate the requirement of any of the expressed receptors in mediating profibrotic effects induced by the SASP LTs, we used 

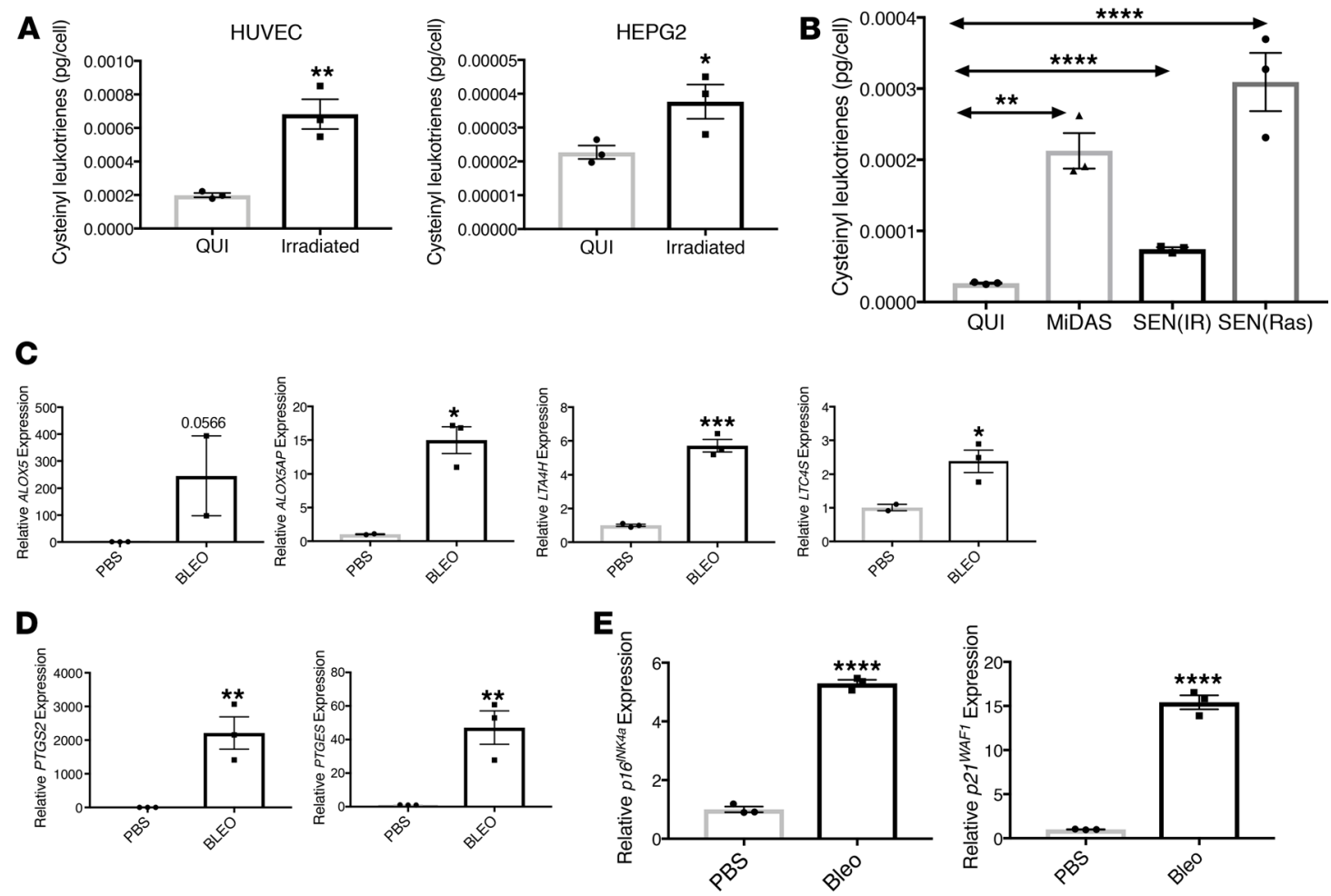

Figure 2. Leukotriene secretion is a common feature of senescent cells. (A) HUVEC and HEPG2 cells were cultured in DMEM + $0.2 \%$ serum (quiescence, QUI) or irradiated (10 Gy, SEN[IR]). The secretion of leukotrienes was assessed measuring the level of cysteinyl leukotriene by ELISA in the conditioned medium 10 days after irradiation. (B) Level of cysteinyl leukotriene from IMR-90 fibroblasts in which senescence was promoted by diverse inducers (MiDAS, mitochondrial dysfunction-associated senescence; irradiation, SEN[IR]; Ras oncogene-induced, SEN [Ras]) and compared with the level from QUI cells. (C-E) IMR-90 were treated with $50 \mu \mathrm{g} / \mathrm{mL}$ bleomycin for 3 hours. Total RNA was isolated, reverse transcribed, and analyzed by qPCR. Signal was normalized to tubulin mRNA. Activation of eicosanoid biosynthesis was assessed by measuring the level of expression of genes encoding leukotriene (C) and prostaglandin (D) biosynthesis enzymes. Senescence was assessed by measurement of increased expression $p 16^{\text {INK } 4 a}$ and $p 21^{\text {WAF1 }}$ mRNA level (E). Data are presented as mean \pm SEM of at least 3 replicates. Statistical analyses were performed using Student's $t$ test $(\mathbf{A}, \mathbf{C}, \mathbf{D}$, and $\mathbf{E})$ or 1 -way ANOVA (B); ${ }^{*} P \leq$ $0.05 ;{ }^{* *} P \leq 0.01 ;{ }^{* *} P \leq 0.001 ;{ }^{* * *} P \leq 0.0001$.

pharmacological antagonist LT receptors LY255283 (a selective, competitive $\mathrm{BLT}_{2}$ receptor antagonist) and montelukast sodium (CysLT1 and GPR17 antagonist). We generated CM from control (day 0) or senescent cells, 10 days after irradiation, and treated naive fibroblasts for 48 hours with CM and LT receptor antagonists (Figure 4A). The activation of COL1A2 gene expression was assessed (Figure 4B). Treatment with vehicles alone didn't affect the level of expression of COL1A2 gene, as expected. Decreased COL1A2 gene expression was only measured when the cells were treated with 10-day $\mathrm{CM}$ and montelukast sodium, in agreement with reports that montelukast antagonizes bleomycin-induced lung fibrosis in mice (34).

To assess whether senescence-associated LTs influence the expression of other SASP factors, senescent fibroblasts were continually treated with the ALOX5 inhibitors Zil or BW. After 10 days, as expected, the level of expression of proinflammatory factors known to be part of the SASP (IL-8, CXCL1, IL-6, IL-1b, IL-1a, CCL2, and VEGF) were upregulated in irradiated senescent cells compared with controls. Treatment with the ALOX5 inhibitors did not affect or moderately decreased the expression level of these genes, suggesting that the LTs secreted by senescent cells have little to no effect on the expression of other proinflammatory factors of the SASP (Figure 5A). Similarly, induction of profibrotic factors such as SERPINE-1, TIMP1, COL1A2, and FIBRONECTIN (FN1) mRNA were unchanged by the presence LT inhibitors (Figure 5B). Furthermore, mRNA expression of receptors for LTB4 (LTBR2) and cysteinyl LTs (CYSLTR2) was lost from senescent fibroblasts (Figure 5C), suggesting that LTs produced by senescent cells are most likely to have paracrine effects rather than autocrine ones.

We also investigated whether senescence-associated LTs promoted senescence in naive fibroblasts by assessing p16 mRNA expression in these cells after 48 hours of treatment with CM collected from 10-day postirradiated fibroblasts (Supplemental Figure 1C). No change in expression was measured; therefore, our data would not support a paracrine effect for $\mathrm{LT}$ in the induction of senescence. 
A

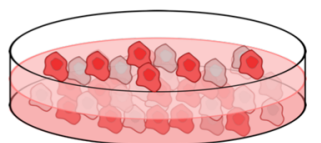

Senescent IMR 90 fibroblasts (10 Gy irradiation, serum-free DMEM ) $+/-50 \mu \mathrm{M}$ Zileution (Alox5 inh, leukotrienes) $+/-1 \mu \mathrm{g} / \mathrm{mL}$ NS-398 (PTGS inh, prostaglandins)

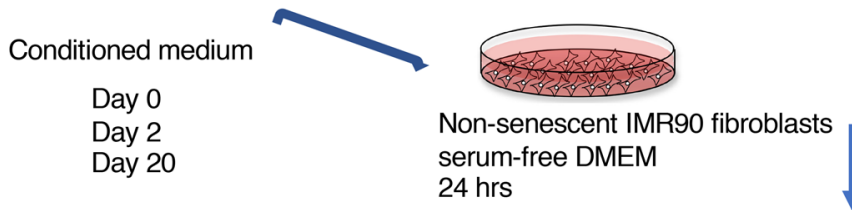

$24 \mathrm{hrs}$
Fibrosis responses col1a2 mRNA aSMA mRNA
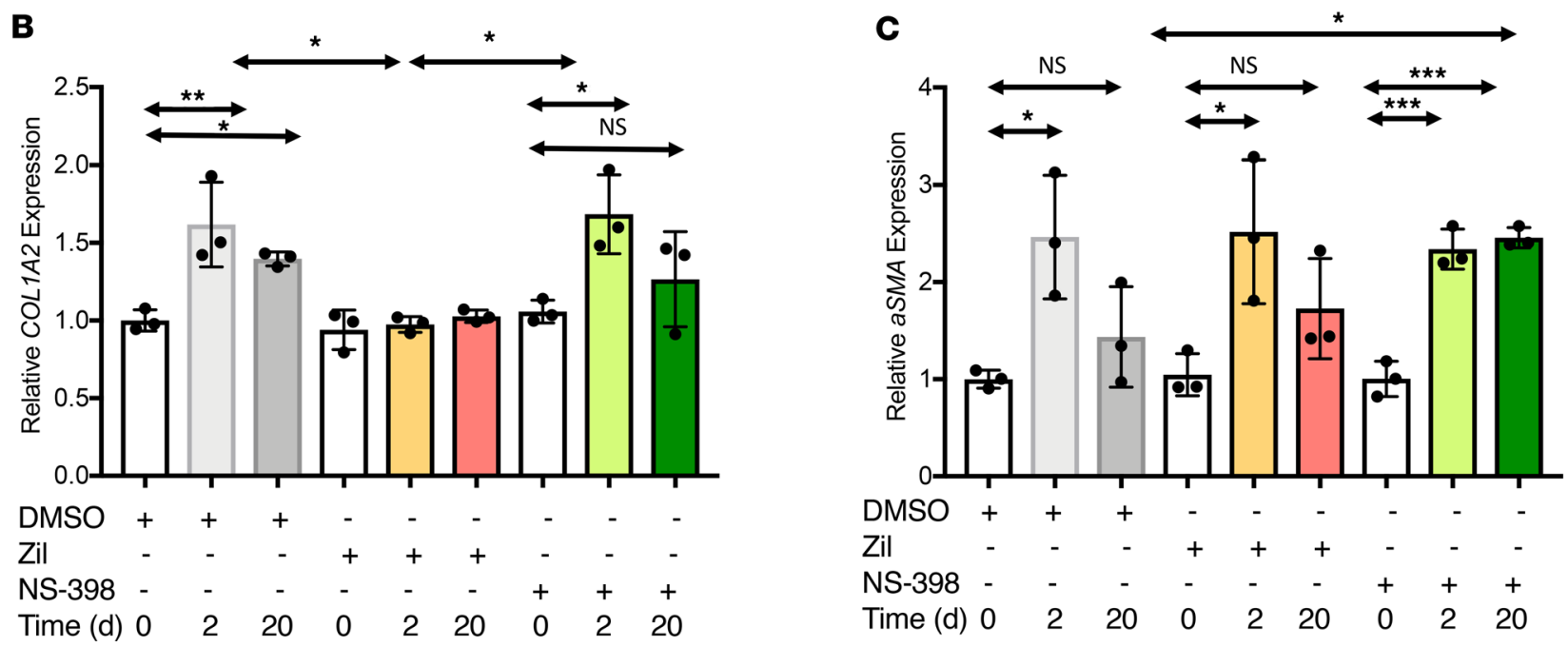

Figure 3. Leukotriene released by senescent cells induces profibrotic responses from naive fibroblasts in vitro. (A) Irradiated human lung fibroblasts (IMR-90) were treated with the ALOX5 inhibitor zileuton (Zi, $50 \mu \mathrm{M})$ or the PTCS inhibitor NS-398 $(1 \mu \mathrm{g} / \mathrm{mL})$ for 24 hours prior to the conditioned medium (CM) collection. CM was collected 2 and 20 days after radiation and applied to nonsenescent IMR-90 fibroblasts for 48 hours. (B and C) Total RNA was isolated, reverse transcribed, and analyzed by quantitative PCR. Signal was normalized to tubulin mRNA. The profibrotic responses were assessed by the expression of COL1A2 (B) and $\alpha-S M A$ mRNA (C). Data are presented as mean \pm SEM of at least 3 replicates. Statistical analyses were performed using 1 -way ANOVA. Asterisk represents the statistical differences calculated by time point. Statistical differences calculated within the same time point group (2 or 20 days); ${ }^{*} P \leq 0.05 ;{ }^{* *} P \leq 0.01$.

Taken together, these data support a model in which early LT synthesis by senescent cells stimulates collagen synthesis and fibrosis, but not senescence, by a paracrine mechanism, whereas the later PG synthesis by senescent cells antagonizes myofibroblast differentiation and collagen expression. In addition, among the senescence-associated LTs, our data indicate that naive fibroblasts could respond to any member of the LT family based on the expression of various receptors. However, based on treatment with antagonists, it is more likely that the cysteinyl LTC4 and LTD4 contribute to the profibrotic effect rather than the noncysteinyl LTB4.

Senescent cells release LTs in the lungs and contribute to fibrosis. To determine whether senescence-associated LT and PG biosynthesis occurs in vivo, and to be consistent with our in vitro data using lung fibroblasts, we asked whether senescent cells were responsible for LT-driven disorders in the lungs. LTs were previously shown to significantly contribute to fibrotic responses in the lungs $(21,35-37)$. Using the classical bleomycin-induced fibrosis model, WT C57BL/6J mice were subsequently treated with vehicle or ABT-263, a BCL2/BCL-XL/ BCL-W inhibitor that selectively eliminates senescent cells in some tissues $(38,39)$. $p 16^{\text {Inkad and }}$ a $21^{\text {Waft }}$ RNA levels increased 14 days after bleomycin injury and were significantly reduced by ABT-263 (Figure 6A).

To assess whether the eicosanoid biosynthesis was affected by the senolytic treatment with ABT-263, we first measured the activation of cPLA2 by determining the level of expression of its phosphorylated form by Western blot. We measured a significant 1.5 -fold expression increased in the bleomycin-injured mice that was reduced by ABT-263 (Figure 6B). Subsequently, the level of mRNA expression of enzymes, ALOX5, LTC4S, PTGS2, PTGDS, and PTGES, involved in eicosanoid biosynthesis was also measured. Their respective mRNA levels were increased in the lungs of bleomycin-injured mice, and elimination of senescent cells with ABT-263 lowered these levels to those of control animals (Figure 6C). Consequently, bleomycin increased the levels of both cysteinyl LTs and PG E2 in the bronchoalveolar lavage fluid (BALF) 21 days after injury, and ABT-263 reduced both levels (Figure 6D). The removal of senescent cells was also 
A

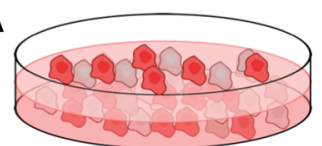

Senescent IMR 90 fibroblasts (10 Gy irradiation, serum-free DMEM)

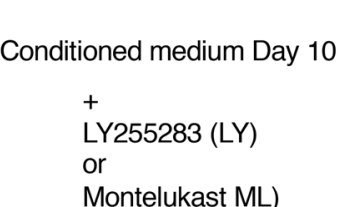

Montelukast ML)

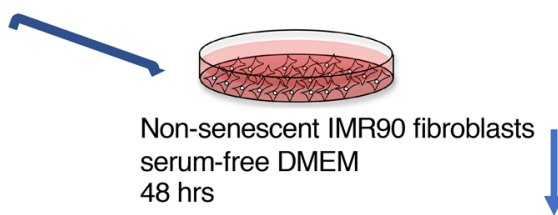

48 hrs
Fibrosis responses col1a2 mRNA
B

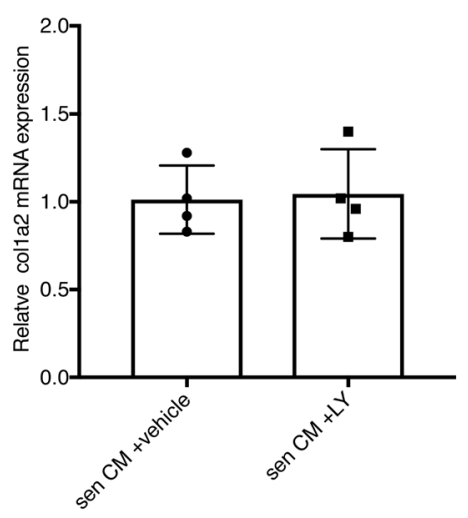

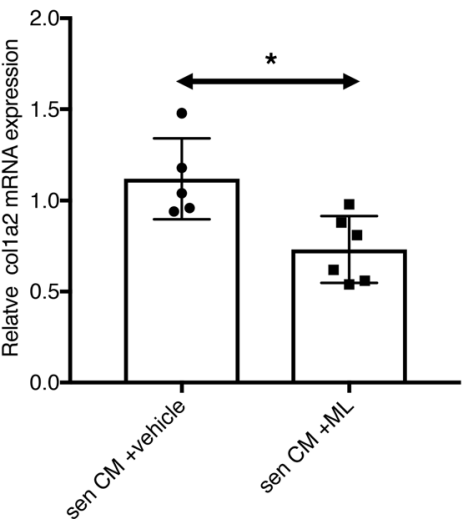

Figure 4. Pharmacological inhibition of the cysteinyl leukotrienes reduces the profibrotic effect of senescent cells on naive fibroblasts in vitro. (A) Schematic representation of conditioned media (CM) studies. The CM of senescent human lung fibroblasts (IMR-90) was collected 10 days after irradiation (10 Gy irradiation), LT antagonists LY255283 (LY, $50 \mathrm{nM}$ ), montelukast sodium (ML, $25 \mathrm{nM}$ ), or vehicle were added to the CM and applied to nonsenescent IMR-90 fibroblasts for 48 hours. Total RNA was isolated, reverse transcribed, and analyzed by quantitative PCR. Signal was normalized to $\beta$-actin mRNA. The inhibitory effect of LT antagonists was assessed by the expression of COL1A2 mRNA. (B) Relative levels of COL1A2 mRNA in cells treated with CM supplemented with either LY (left) or ML (right). Data are presented as mean \pm SEM of at least 4 replicates. Statistical analyses were performed using unpaired Student's $t$ test; ${ }^{*} P \leq 0.05$.

significantly associated with an attenuation of collagen content assessed by histological staining with Picrosirius red (Figure 6E) and measurement of hydroxyproline (Figure 6F) 21 days after injury. Furthermore, ABT-263 treatment attenuated the increase in collagen mRNA (Col3a1 and Col4a1, Supplemental Figure 2).

To confirm that the attenuation of cysteinyl LT levels in BAL after ABT-263 treatment was the result of deletion of $p 16^{\text {Inkta+}}$ cells, we undertook 2 separated approaches. First, as one of the major cell populations secreting LTs, CD45 cells were isolated from bleomycin-injured lungs 14 days after injection and were treated with ABT-263 for 48 hours. Our data indicate that the level of expression of LT or PG biosynthesis enzymes in $\mathrm{CD}^{4} 5^{+}$cells was not directly affected by ABT-263 treatment (Figure 7A). In addition, the percentage of $\mathrm{CD}^{4} 5^{+}$cells in the lungs 14 days after bleomycin injury were not affected by ABT-263 (Figure 7B). Second, $p 16^{\text {Inkta }}-3 \mathrm{MR}$ mice were injured with bleomycin and then treated with ganciclovir (GCV). p16 $6^{\text {Ink4a }}-3 \mathrm{MR}$ mice contain a transgene that permits the detection and selective killing of $p 16^{\text {Ink4a+ }}$ senescent cells by administering GCV, an otherwise innocuous drug (10). Our data indicate that deletion of $p 16^{\text {Ink4a+ }}$ cells resulted in reduced collagen deposition, as well as reduced level of cysteinyl LT in the BAL of the GCV-treated injured $p 16^{\text {Inkta }}$-3MR to a similar level as the level after ABT-263 treatment (Figure 7, C-E).

These data indicate that removal of senescent cells is associated with both reduced eicosanoid biosynthesis and collagen deposition, consistent with the hypothesis that the senescent cells are an underlying driver of bleomycin-induced pulmonary fibrosis due to the secretion of LTs as part of their SASP or by inducing LT expression in other cells via a paracrine effect.

An interesting feature of the bleomycin-induced pulmonary fibrosis mouse model is that it resolves over time $(40,41)$, suggesting that either senescent cells are eventually cleared or the fibrosis-promoting properties of senescent cells change over time. We therefore analyzed $\mathrm{p} 16^{\text {Ink4a }}$ and collagen (COL1A2) mRNA levels over a 42-day period following bleomycin-induced injury. Notably, although p16 $6^{\text {Ink4a }}$ expression increased over 14 days after injury, peaked 30 days after injury, and remained elevated through day 42 (Figure $7 \mathrm{~F}$, green line), collagen expression peaked at 14 days after injury but, thereafter, progressively declined (Figure 7F, orange line). These data suggest that the fibrosis-inducing properties of senescent cells change over time. Because we observed an early rise in LT synthases expression (Figure 1G), followed by a later rise in PG synthases expression (Figure 1H) in cultured 

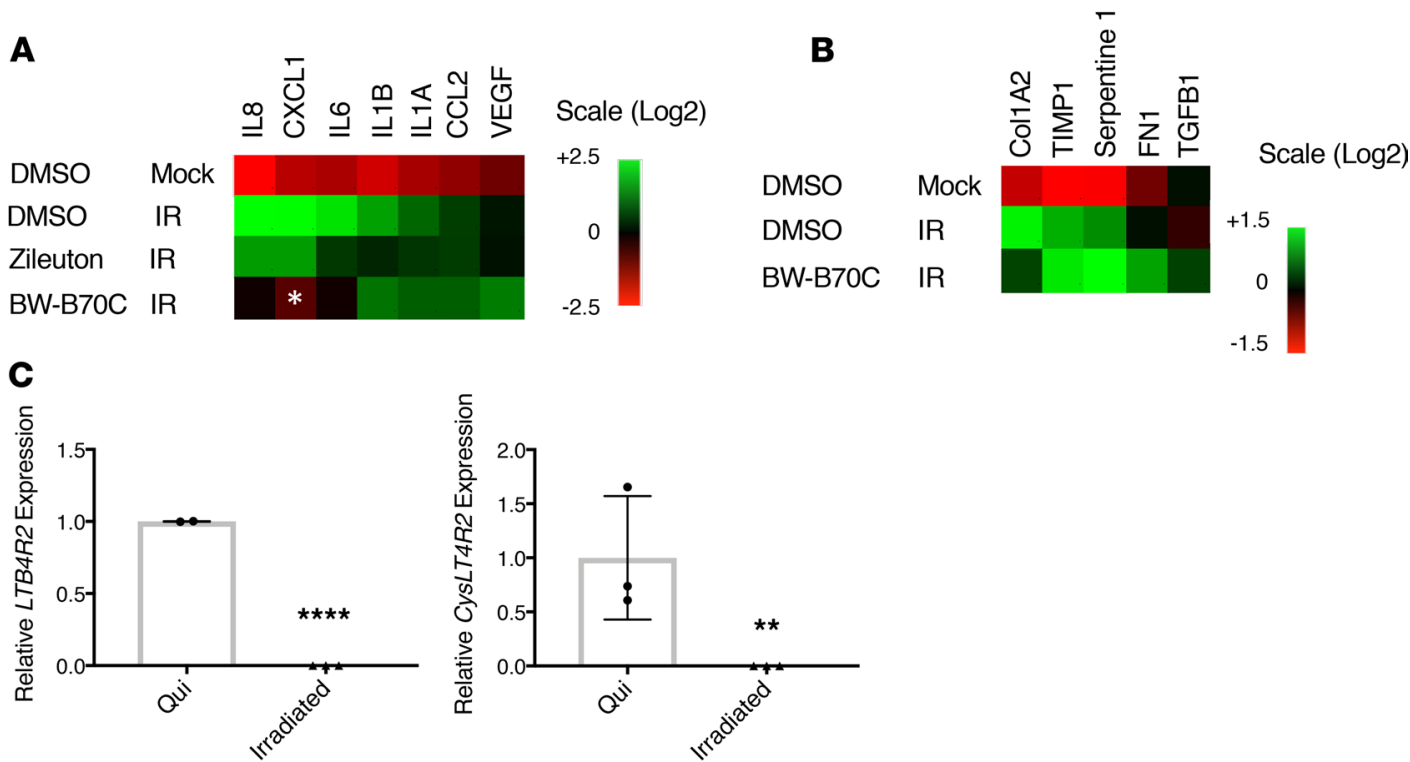

Figure 5. Inhibition of ALOX5 activity modulates the expression of proinflammatory SASP factors. Irradiated human lung fibroblasts (IMR-90) were treated with the ALOX5 inhibitor BW-B70C (BW, $10 \mu \mathrm{M})$ for 10 days. Total RNA was isolated, reverse transcribed, and analyzed by quantitative PCR. Signal was normalized to tubulin mRNA. (A and B) Heatmap representation of the level of expression mRNA levels of proinflammatory SASP components (A) and the level of expression mRNA levels of profibrotic SASP components (B). (C) Decreased mRNA expression of genes encoding LT receptors in senescent cells 10 days after irradiation. Data are presented as mean \pm SEM of at least 3 replicates. Statistical analyses of $\mathbf{A}$ and $\mathbf{B}$ were performed using 2-way ANOVA, and significance was determined for $\mathbf{C}$ by 2-tailed Student's $t$ tests with Welch's correction. ${ }^{* *} P \leq 0.01 ;{ }^{* * *} P \leq 0.0001$.

cells, we tested whether a similar pattern developed in vivo. Indeed, bleomycin injury caused an early spike in whole lung ALOX5 expression (Figure 7G), followed by a progressive rise in the expression of PTGDS, which synthesizes PGD2 (Figure 7G), consistent with a switch in senescent cell phenotype from profibrotic to antifibrotic.

Senescent IPF fibroblasts synthesize LTs but fail to produce PGs. Previous reports indicate that fibroblasts from IPF patients fail to elevate PG synthesis (42) or fail to respond to PG treatment (43). To determine whether senescent IPF fibroblasts synthesize LTs and PGs in a similar fashion to senescent normal lung fibroblasts, we compared mRNA levels of ALOX5, PTGS2, PTGDS, and PTGES in control and radiation-induced senescent fibroblasts isolated from normal and IPF lungs. As expected at baseline, IPF lung fibroblasts expressed high levels of $A L O X 5$ mRNA compared with normal lung fibroblasts. However, even though ALOX5 mRNA expression increased upon irradiation-induced senescence in both cell types, IPF fibroblasts still expressed significantly higher levels of ALOX5 mRNA (Figure 8A). Compared with nonirradiated cells, senescent normal lung fibroblasts increased expression of mRNA of the PG synthases PTGS2 and PTGD. This induction of PG biosynthesis enzyme expression is also measured in senescent IPF fibroblasts but to a much lesser extend (Figure 8A). These data suggest that IPF patients respond differently to senescence-inducing stimuli with regard to PG synthesis. Senescent cells, as defined as $p 16^{\text {Inkata }}$ cells, $\mathrm{ALOX5}^{+}$cells, and dual-positive cells, are detected in IPF lung in greater numbers than in normal lungs (Figure $8, \mathrm{~B}$ and C). Overall, the $p 16^{\text {Inktat }}$ cells represented an average $30 \%$ of total cells in the IPF lungs, of which $50 \%$ expressed ALOX5 (Figure 8C). Because several cell types, mainly type II epithelial cells and fibroblasts, have been previously reported to undergo senescence in IPF lungs (44), we costained $\mathrm{ALOX}^{+}$cells with marker for type II epithelial cells (surfactant protein C) and for fibroblasts (vimentin). Very few ALOX $5^{+}$cells, less than $3 \%$, were detected in the $\mathrm{SPC}^{+}$population (Figure 8, D and E). However, $23.3 \% \pm 5.7 \%$ of $\mathrm{ALOX}^{+}$cells also costained for vimentin (Figure 8, D and E). Despite this staining, we did not detect the expression of ALOX5 in myofibroblast cells, as defined as $\alpha-\mathrm{SMA}^{+}$(data not shown), indicating that the producers of LTs are a distinct population of lung fibroblasts. Together, our experiments point to senescent lung fibroblasts as a key source of profibrotic LTs during lung fibrosis. 
A

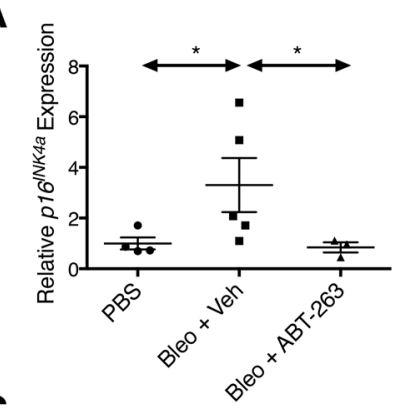

C
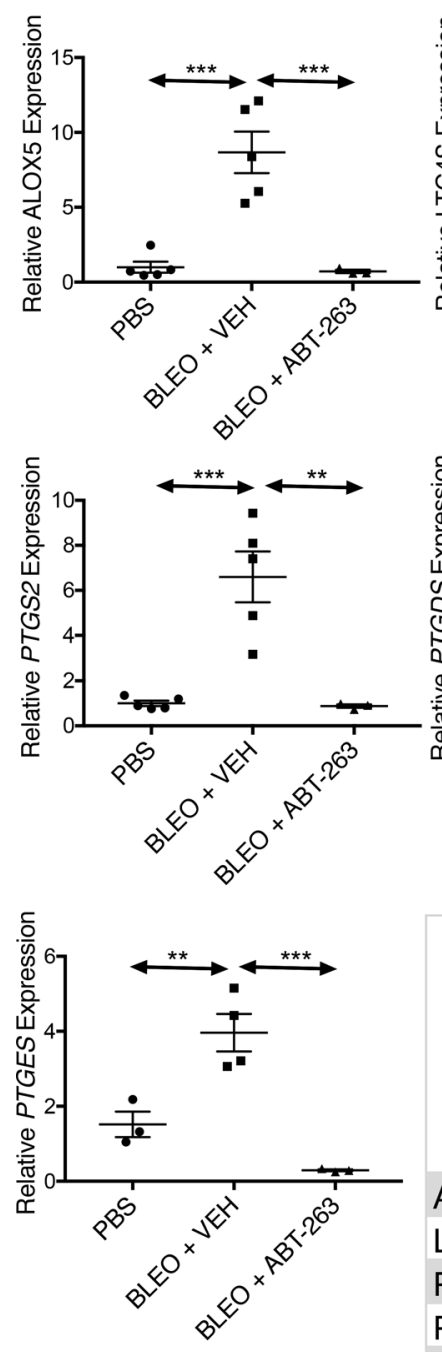

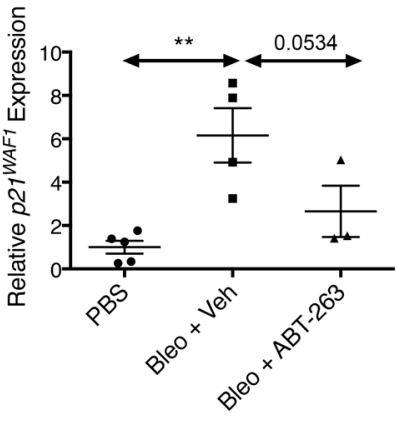

B
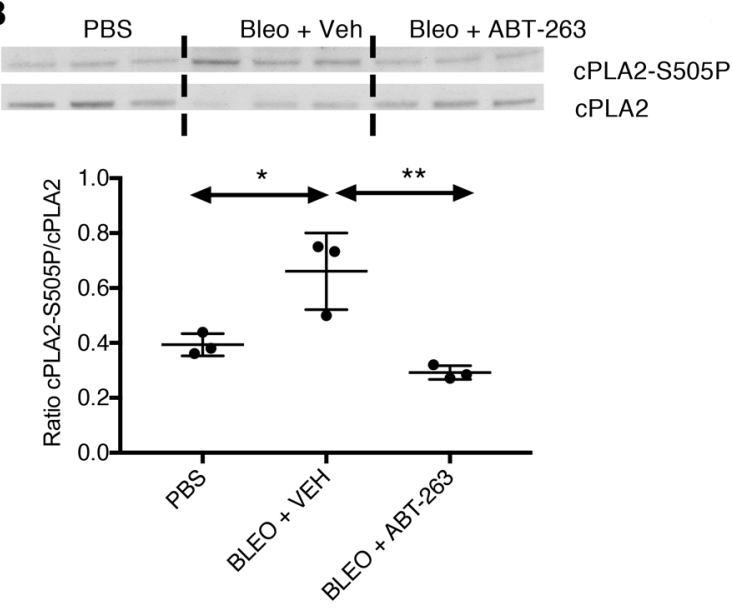

D

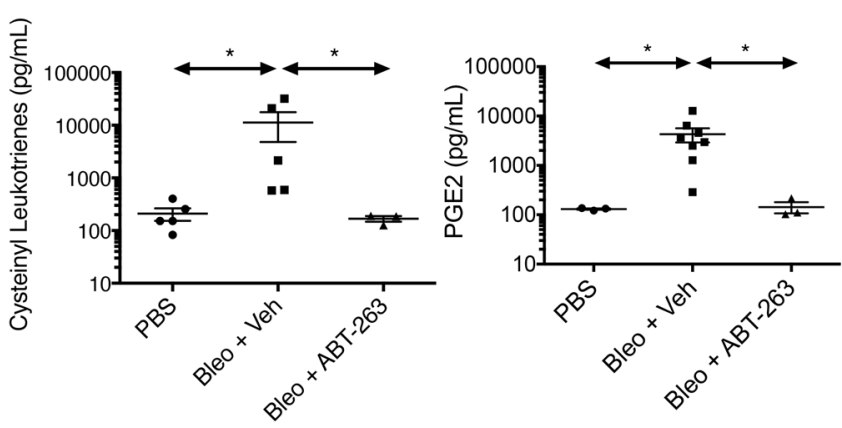

E PBS

Bleo + Veh

Bleo +ABT 263
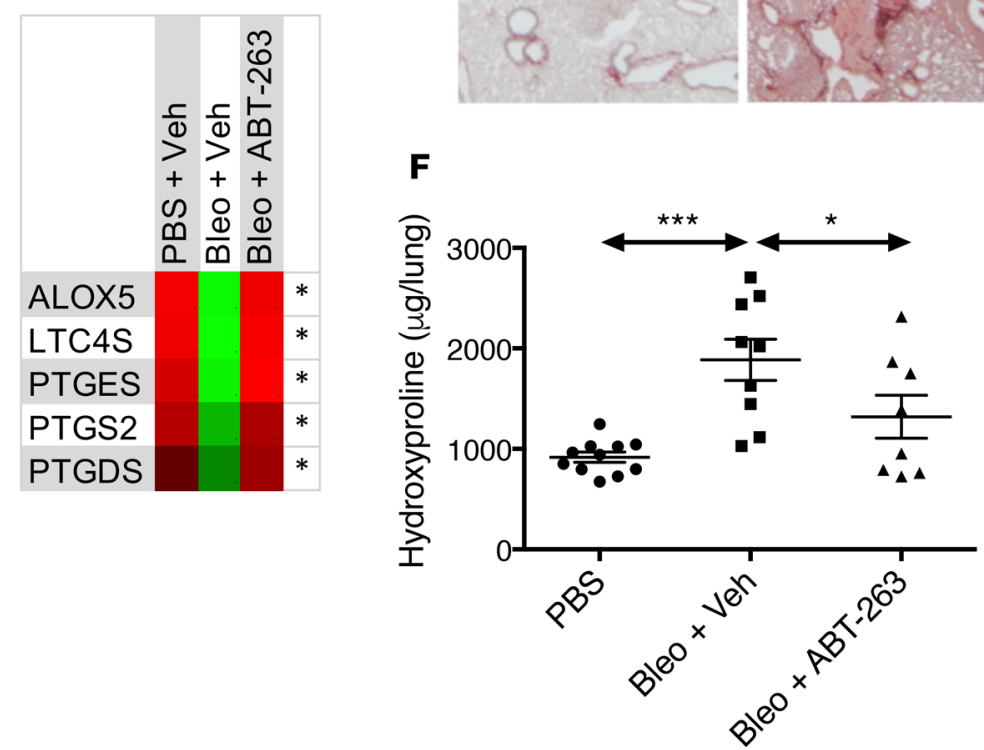
Figure 6. Senescence-associated leukotriene synthesis promotes pulmonary fibrosis. WT C57BL/6] mice received a single intratracheal injection of PBS (vehicle control) or bleomycin (Bleo, $1.9 \mathrm{U} / \mathrm{kg}$ ). Mice received, from days 7-14,vehicle (Veh) or $50 \mathrm{mg} / \mathrm{kg} / \mathrm{day}$ ABT-263 by gavage. (A) Level of $p 16^{I N K 4 a}$ and p21 WAF1 mRNA levels normalized to tubulin mRNA. (B) Activation of cytosolic phospholipase A2 (cPLA2) was determined by calculating the ratio of the level of expression of phosphorylated cPLA2 to the level of expression of total cPLA2. Representative pictures of Western blot of lung lysates collected day 14 after the bleomycin injury for the expression of the phosphorylated form of cPLA2, CPLA2-S505P, and nonphosphorylated form cPLA2. $n=3$ lysates per group. (C) Level of expression of mRNA of gene encoding enzymes of the leukotriene (ALOX5 and LTC4S) and the prostaglandins (PTCDS, PTCS2, and PTGES) biosynthesis pathways was measured by qPCR normalized to tubulin mRNA, in samples collected 14 days after bleomycin injury. Data are presented as dot plot graphs or heatmap. (D) Lipids were extracted from broncho-alveolar lavage fluid (BALF) collected 14 days after bleomycin injury and treatment with vehicle or ABT-263. BALF lipid content was analyzed for cysteinyl leukotrienes and for PGE2 by ELISA. (E) Representative pictures of Picrosirius red staining of lungs collected 21 days after bleomycin injury and treatment with vehicle or ABT-263. Original magnification, $\times 100$. (F) Hydroxyproline levels obtained using the right lung lobes of mice, 21 days after bleomycin injury and treatment with vehicle or ABT-263. Unless stated otherwise, lung and BALF from at least 5 PBS-treated mice, 5 bleomycin + vehicle-treated mice, and 3 bleomycin + ABT-263 mice were analyzed. Statistical analyses were performed using 1-way ANOVA test. ${ }^{*} P \leq 0.05 ;{ }^{*} P \leq 0.01$.

\section{Discussion}

The contributing role of senescent cells to physiological or pathological processes is mainly attributed to the release of secreted factors grouped as SASP. Thus far, the SASP has been defined as a complex set of secreted proteins that are variable depending on the cell type and driver of senescence $(10,16,45)$. Here, we show that senescent cells also synthesize a number of eicosanoids and, in particular, LTs. The expression of LTs appears to be ubiquitous, regardless of the modality of senescence induction and - to some extent - of the cell types when tested in vitro. However, the pattern of eicosanoid expression, as reflected by dynamic changes in the mRNA levels of ALOX5 and COX2, is a time-dependent process (Figure 1). It is commonly accepted that the phenotype of senescent cells is highly heterogeneous, depending upon specific stresses and cell types (46). More recently, the transcriptomic signature of fibroblasts, melanocytes, and keratinocytes were obtained 4,10 , and 20 days after ionizing radiation. Hernandez-Segura et al. demonstrated that, among the 55 genes of the senescence core signature that they have previously identified, 13 genes were differentially regulated at every time point and in every cell type (46). In this study, the expression of biosynthetic enzymes of the LTs and PGs is clearly time dependent. This suggest that the regulation of the expression of SASP factors is very tight. These findings emphasize the need to include time as a variable when studying the relevance and the role of senescent cells in any pathology. As LTs are released, they moderately modify the expression of some but not all SASP elements. In parallel, LT receptor expression declined over time, suggesting an autocrine control from SASP factors. Overall, dynamic changes in eicosanoid expression over time superimposes another layer of complexity on the evolution of the SASP. This complexity may explain some of the disparate findings on the physiological impact of senescence on normal and pathological processes, including the controversial role of senescent cells in fibrosis. Senescent cells appear to have a negative impact in lung fibrosis (Figure 5 and refs. 5, 7, 13-15, 47) in contrast with a positive effect in CCL4-induced liver fibrosis or in wound healing $(10,48)$. This complexity underscores the challenge in developing senolytics as therapeutic agents.

In a recent model of skin wound healing, senescent fibroblasts were detected 3-6 days after injury. Interestingly, these senescent fibroblasts were found to be critical to normal wound healing, as their deletion via the p16-3MR transgenic system delayed wound repair (1). In other contexts, however, the induction of a SASP could be expected to promote tissue injury. In healthy older individuals, for example, protease secretion by senescent cells is believed to facilitate the enlargement of airway spaces in a mechanism similar to tobacco-related emphysema (49). These temporal changes of the SASP could also explain the deceptive contradiction in the role of senescent cells in wound healing and fibrosis. It has been already demonstrated that the removal of senescent cells in various animal models using genetic mouse models or pharmacology intervention alleviates the development of fibrosis $(13,38,39,47)$.

As senolytic therapeutic approaches emerge, it is important to better understand these dynamic and temporal changes of the SASP, as the role of the senescent cells may change during the course of the disease.

LTs and PGs have been implicated in the pathobiology of pulmonary fibrosis for more than 2 decades (27). LTs synthesis via ALOX5 is constitutively activated in IPF (50), and genetic deletion of ALOX5 attenuates development of fibrosis in a murine model of lung injury (35). Conversely, inhibition of PG synthesis via either genetic deletion or inhibition of COX2 exacerbates the development of pulmonary fibrosis in mice (22), and PGE2 and COX2 levels decline in patients with IPF $(24,51,52)$. Notably, genetic deletion of ALOX5 not only results in loss of LT synthesis following lung damage, but also in elevation of PGE2 levels (35). The source of the LTs has been believed to be mainly macrophages; how- 
A

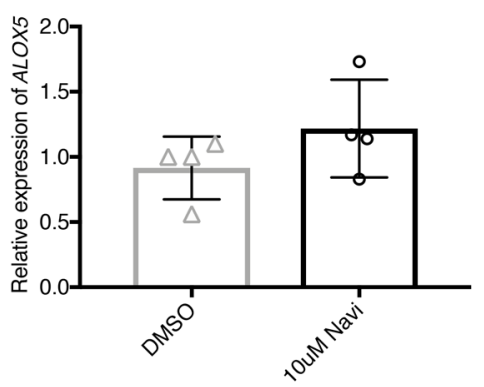

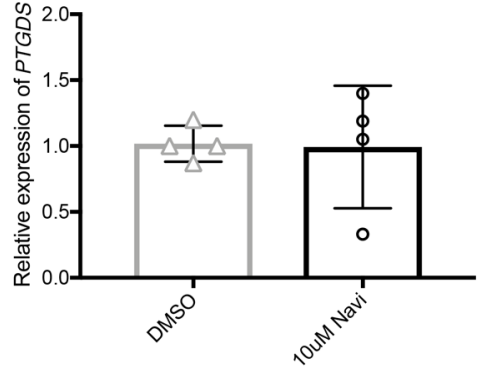

B Navi 1

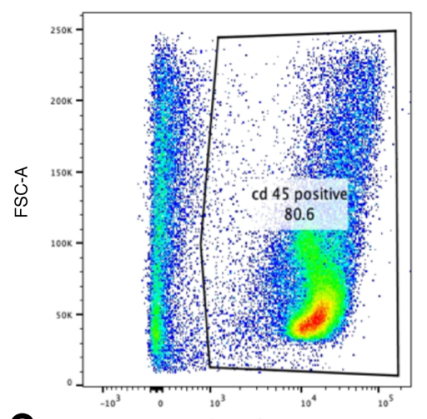

C

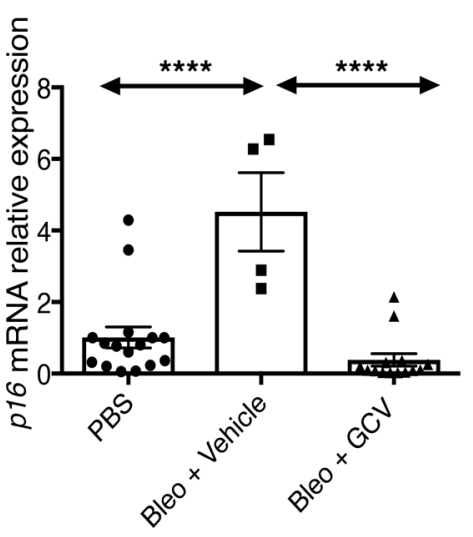

$\mathbf{F}$

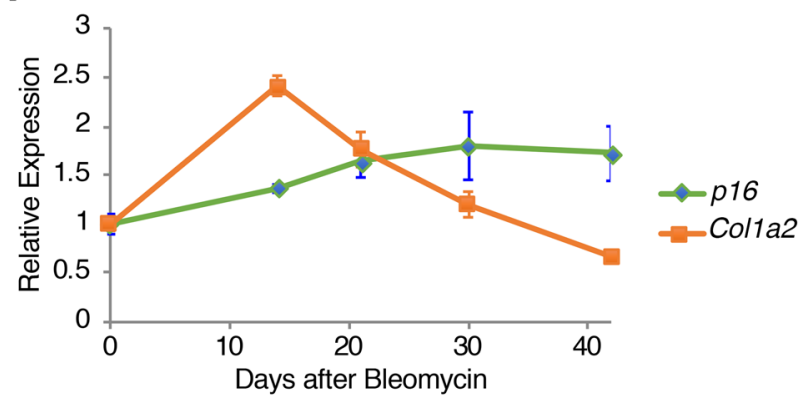

D
Vehicle 1
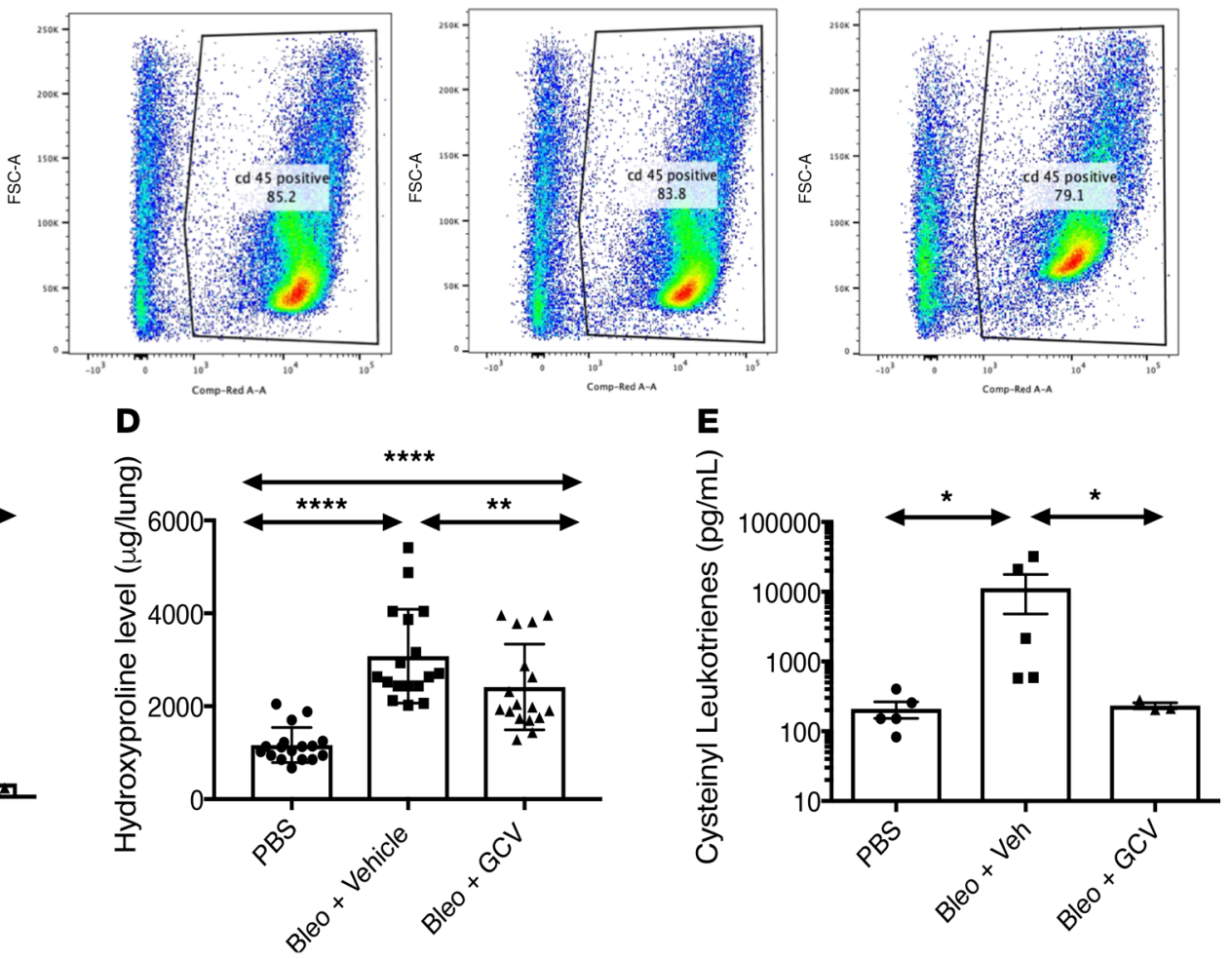

G

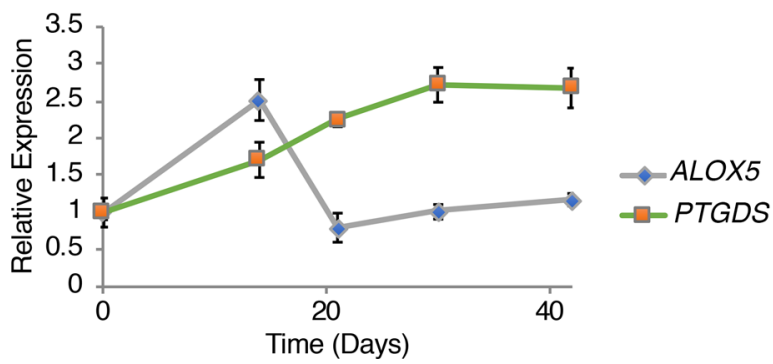

Figure 7. Temporal changes in eicosanoid biosynthesis reveal pro- and antifibrotic activities of senescent cells. (A) Isolated CD45 ${ }^{+}$cells from $14-d a y$ bleomycin-injured lungs were treated with DMSO and $10 \mu \mathrm{M}$ ABT-263 for 48 hours. Analysis of mRNA expression of LT and PC biosynthesis enzymes was performed by qPCR. (B) Analysis by flow cytometry of percent of $C D 45^{+}$cells isolated from 14-day bleomycin-injured lungs treated ABT-263 or vehicle for 7 days. (C and D) p16 $6^{\operatorname{lnk} 4 a}$-3MR mice received a single intratracheal injection of PBS (vehicle control) or bleomycin (Bleo, 1.9U/kg). (C) Level of $p 16^{1 N k 4 a} \mathrm{mRNA}$ levels normalized to tubulin mRNA. (D) Hydroxyproline levels obtained using the right lung lobes of mice, 21 days after bleomycin injury. (E) Lipids were extracted from broncho-alveolar lavage fluid (BALF) collected 21 days after bleomycin injury. BALF lipid content was analyzed for cysteinyl leukotrienes by ELISA. (E) Whole lung tissues were collected 14, 21, and 42 days after PBS or bleomycin intratracheal injection for RNA extraction (7 mice per group) and analyzed by qPCR. (F) mRNA levels of p16 ${ }^{I N K 4 a}$ (p16, green line) and collagen (COL1A2, orange line), normalized to tubulin mRNA. (C) mRNA levels of ALOX5 (gray line) and PTCDS (green line) normalized to tubulin mRNA. Statistical analyses were performed using Student's $t$ test (A), 1-way ANOVA (C, D, and E), or individual 2-tailed unpaired Student's $t$ test. ${ }^{*} P \leq 0.05 ;{ }^{* *} P \leq 0.01 ;{ }^{* * *} P \leq 0.001$. 
A
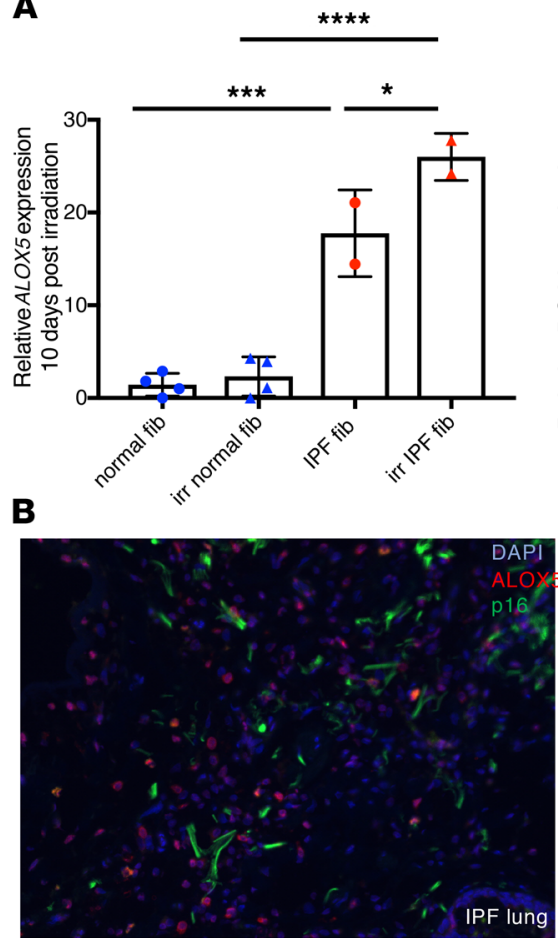

C
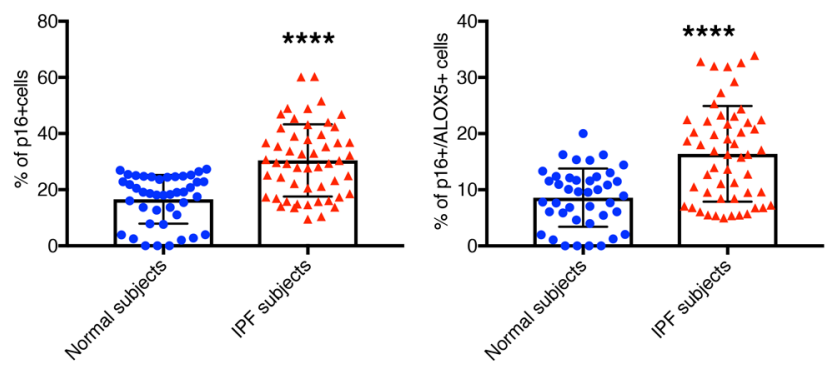

D
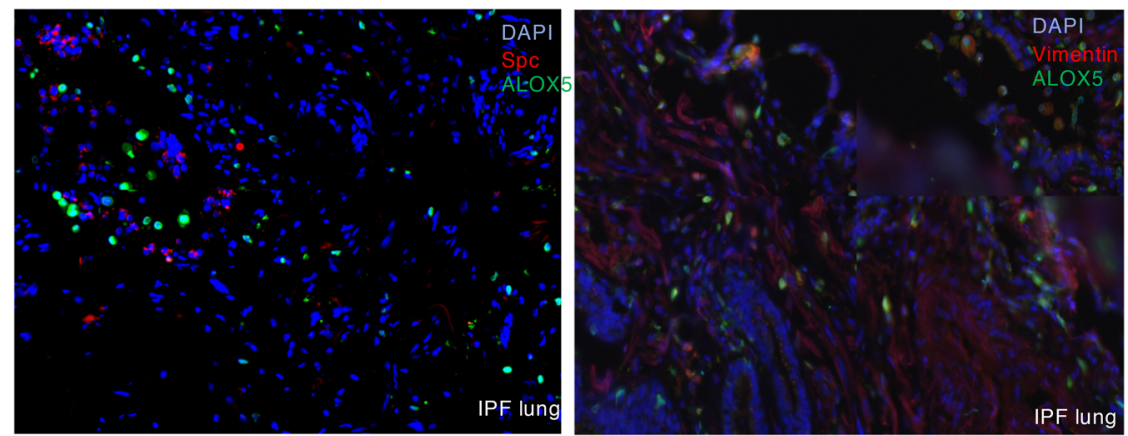

\section{E}

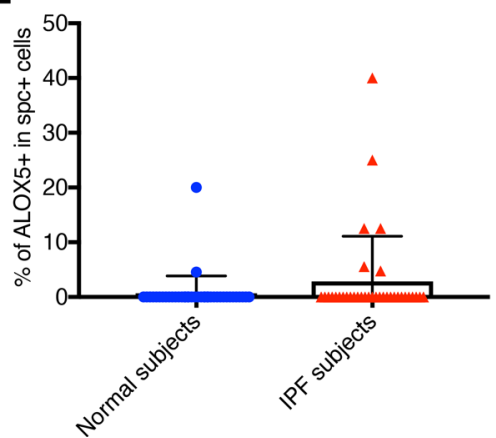

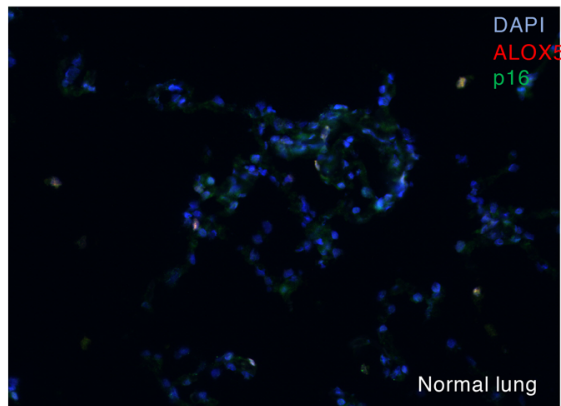
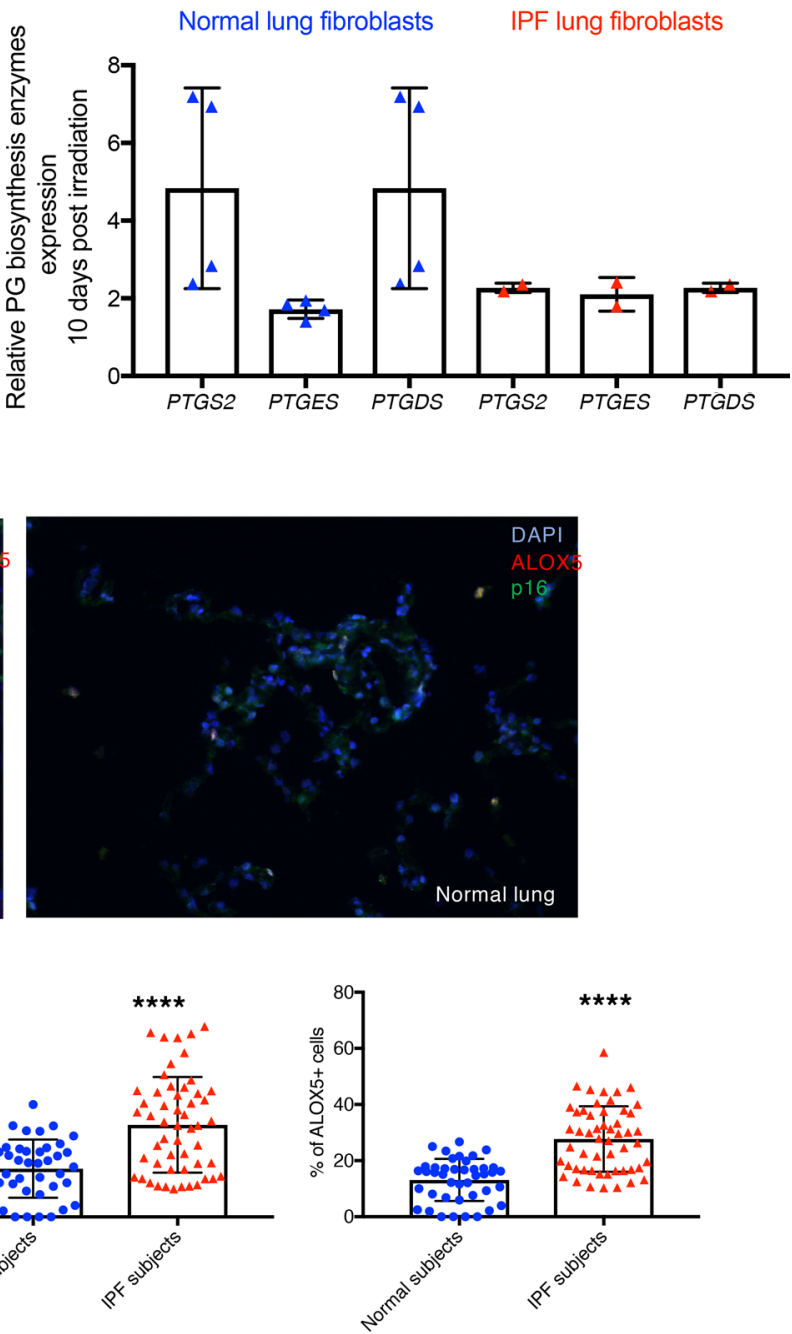

Figure 8. Human senescent fibroblasts express ALOX5 but not COX2 in IPF lungs. (A) Primary fibroblasts isolated from normal and IPF lungs $(n=2)$ were induced to senescence by ionizing radiation (10 Gy, irradiation). Total RNA was collected 10 days later, reverse transcribed, and analyzed by qPCR for expression levels of ALOX5, PTCS2, PTCDS, and PTCES MRNA, normalized to L19 mRNA. Data are presented as means \pm SEM for 3 independent experiments. (B) Representative pictures of immunodetection of senescent cells ( $p 16^{\text {Ink } k a+)}$ coexpressing ALOX5 in normal and IPF lungs. (C) Cell count of senescent $p 16^{\text {Inkaa+ }}$ cells and $\mathrm{ALOXS}^{+}$cells detected by immunofluorescence in normal and IPF lungs. (D) Representative pictures of immunodetection of type II epithelial cells (SPC+) and fibroblasts (Vimentin+) coexpressing ALOX5 in normal and IPF Iungs. (E) Cell count of senescent $\mathrm{SPC}^{+}$cells, Vimentin ${ }^{+}$cells, and ALOX5+ cells detected by immunofluorescence in normal and IPF lungs. $n=2$ independent subjects per group. Each dot represents number of cells per field, and bar graph represents means \pm SEM. Statistical analysis was perform using 1-way ANOVA Holm-Sidak's multiple comparisons test (A) or unpaired Student's $t$ test ( $\mathbf{C}$ and E). ${ }^{*} P \leq 0.05 ;{ }^{* * *} P \leq 0.001 ;{ }^{* * *} P$ $\leq 0.0001$. Original magnification, $\times 200$. 
ever, in the recent years, other cells have emerged as inflammatory cells and, among them, the senescence cells. Quantification of the $p 16^{\text {Ink4a }} /$ ALOX5-double positive cells in IPF tissues as roughly $50 \%$ of all the ALOX 5 cells, and their morphological distinction from macrophages indicates a significant contribution of senescent structural cells to the eicosanoid profile of IPF lungs (Figure 7). Here, we demonstrate that removal of senescent cells using ABT-263 that targets 3 of the essential prosurvival players, BCL-2, BCL-W, and BCL-XL, is not only associated with reduced fibrosis, but also suppression of the LT biosynthesis enzyme expression and secretion in the BAL of cysteinyl LTs. It remains to be determined if senolytics would have similar effects in IPF patients.

Evidence presented in our data (Figures 2-6) indicate that senescent cells may contribute to the development of fibrosis due to the secretion of LTs. Naive fibroblasts expressed a wide variety of LT receptors. However, our data indicate that the profibrotic effects of the LT-enriched SASP may be associated with the presence of cysteinyl LTs in the SASP and the expression of CysTL1/L2 on the surface of the responding cells. This receptor/ligand combination observed here is not surprising, as the roles of LTC4 and LTD4, as well as the CysTL1/L2 receptors, in the regulation of collagen expression by fibroblasts are well established and characterized $(36,53)$. Senescence-associated LT synthesis promoted the expression of profibrotic genes implicated in pulmonary fibrosis in naive fibroblasts (Figure 3). However, over time, the composition of the LTs and PGs in the SASP evolved from being profibrotic to antifibrotic in vitro (Figures 3 and 4). This evolution in vitro is mirrored by the pattern of eicosanoid expression over time in vivo in the bleomycin model, a timing that corresponds well with the ultimate resolution of the fibrosis in the lungs. If these associations are causal, then there are implications for IPF. Our data suggest that IPF progression might result, in part, from an altered senescence response - one that promotes fibrosis via LT release but does not resolve because of the inability of senescent IPF fibroblasts to express COX2 and secrete antifibrotic PGs. The inability of IPF senescent fibroblasts to secrete PGs could explain, at least in part, why the maintenance of senescent cells in the lungs is associated with increased fibrosis, whereas - in other organs, such as the skin or liver - the senescent cells eventually express PGs and attenuate fibrosis.

\section{Methods}

\section{In vitro studies}

Human fetal lung fibroblasts (IMR-90, American Tissue Culture Collection; ATCC) were cultured in DMEM (Thermo Fisher Scientific) supplemented with 10\% FBS (Thermo Fisher Scientific) and penicillin/ streptomycin (Thermo Fisher Scientific). HUVEC (ATCC) and HEPG2 (ATCC) were cultured based on manufacturer's instructions. Normal and IPF fibroblasts were explanted from tissue provided by UCSF Interstitial Lung Disease Blood and Tissue Repository. Quiescence was induced after the cells reached confluence by replacing culture media with media containing $0.2 \%$ FBS. Cells were cultured at $3 \% \mathrm{O}_{2}$ and were mycoplasma free. Inhibition of eicosanoid biosynthesis was performed by adding DMSO (vehicle control), NS-398 (1 $\mu \mathrm{g} / \mathrm{mL}$, Cayman Chemical), BW (10 $\mu \mathrm{M}$, Cayman Chemical), or Zil (50 $\mu \mathrm{M}$, Cayman Chemical) to the serum-free media. Inhibition of LT activity was performed by supplementing CM with LY 255283 (50 nM, Tocris) or montelukast sodium (25 nM, Tocris).

\section{Senescence induction}

Ionizing radiation. Senescence was induced by $10 \mathrm{~Gy}$ X-irradiation. Nonsenescent controls (proliferating or quiescent) were placed in the irradiator for an identical period of time but without irradiation (mock controls).

Mitochondrial dysfunction associated senescence. Mitochondrial dysfunction associated senescence (MiDAS) was induced by depleting mitochondrial DNA in the presence of $100 \mathrm{ng} / \mathrm{mL}$ Ethidium bromide (Sigma-Aldrich) $100 \mu \mathrm{g} / \mathrm{mL}$ sodium pyruvate, and $50 \mu \mathrm{g} / \mathrm{mL}$ uridine (Sigma-Aldrich) for 2 months, followed by removal of pyruvate, as described in ref. 31 .

Bleomycin-induced senescence. Fibroblasts were treated with bleomycin $(50 \mu \mathrm{g} / \mathrm{mL}$, Calbiochem) for 3 hours. Bleomycin treatments were administered at atmospheric oxygen. After 3 hours, cells were washed and media was replaced with bleomycin-free media, cultured at $3 \% \mathrm{O}_{2}$ for 8 days before analysis. Oncogene-induced senescence was induced using lentiviral-mediated overexpression of HRAS ${ }^{\mathrm{v} 12}$, as described (31).

Generation of CM. CM were generated by culturing cells in serum-free DMEM supplemented with penicillin/streptomycin and eicosanoid inhibitors for 24 hours before harvest either 2 or 20 days after irradiation. 
Day 0 cells were sham irradiated. Where indicated, CM were supplemented with $5 \mathrm{ng} / \mathrm{mL}$ TGF- $\beta$ (R\&D Systems) or carrier (BSA). A total of 200,000 cell equivalents/mL of CM with or without TGF- $\beta$ was used to stimulate serum-starved naive nonsenescent IMR-90 fibroblasts for 24 hours prior to isolation of RNA.

\section{Gene expression analysis}

RNA was extracted from cells or tissues using commercially available kits (Isolate II, Bioline for cells; Direct-zol, Zymo for tissues) according to the manufacturer's instructions. cDNA synthesis was performed using a High Capacity cDNA Reverse Transcription Kit (Thermo Fisher Scientific) according to the manufacturer's instructions. Quantitative PCR was performed on a LightCycler 480 II (Roche Diagnostics) using primers and probes designed for the Universal Probe Library and https://pga.mgh.harvard.edu/ primerbank (see list in Supplemental Methods).

\section{Level of expression of protein}

Detection of the activation of cPLA2, p38, and ALOX5 by immunoblot. Cells were lysed in 5\% SDS in $10 \mathrm{mM}$ Tris, $\mathrm{pH} 7.4$, and protein content was determined by BCA assay (Pierce). A total of $20 \mu \mathrm{g}$ protein was separated by electrophoresis and transferred to PVDF membranes. Membranes were blocked in TBST + 5\% BSA (Sigma-Aldrich), incubated overnight with primary antibody, washed in TBST, incubated with HRP-conjugated secondary antibody for 30 minutes, and visualized by chemiluminescence. Densitometry was quantified using NIH ImageJ software. Antibodies used included cPLA2 polyclonal antibody (catalog 2832), phospho-cPLA2 polyclonal antibody (catalog 2831), p38 polyclonal antibody (catalog 9212), ALOX5 monoclonal antibody (C49G1, catalog 3289), and phospho-ALOX5 polyclonal antibody (catalog 3748), purchased from Cell Signaling Technology. Phospho-p38 (catalog p190-1802), $\beta$-actin monoclonal antibody (catalog A5442, clone AC-15), and tubulin (catalog ab6046) polyclonal antibodies were purchased from PhosphoSolutions, Sigma-Aldrich, and Abcam, respectively.

IL-6 ELISA. A total of $3 \times 10^{4}$ cells in 12-well plates were treated as indicated and were cultured in $0.5-1$ $\mathrm{mL}$ serum-free DMEM for 24 hours. CM were collected and clarified at 2,000 $g$ for 10 minutes. Supernatants were transferred to a tube; cells were trypsinized and counted. CM $(2.5 \mu \mathrm{L})$ were analyzed by bead-based ELISAs (AlphaLISA, Perkin-Elmer) as instructed by the manufacturer and normalized to cell number.

\section{Detection of LT}

Cysteinyl LT level was measured in the CM or BALF by ELISA (Cysteinyl Leukotriene, Cayman Chemical; Amersham Leukotriene C4/D4/E4 Biotrak, GE Healthcare) according to the manufacturer's instructions. The concentration of cysteinyl LT in the CM was then normalized to the cell number. LT B4 levels were assessed in cell lysate by mass spectrometry as previously described (54).

\section{In vivo studies}

To induce pulmonary fibrosis, 8- to 10 -week-old male C57BL/6J (the Jackson Laboratory) or $p 16^{\text {Inkta }}-3 \mathrm{MR}$ mice (in house) were given $2 \mathrm{U} / \mathrm{kg}$ body weight of PBS (control group) or bleomycin (Sigma-Aldrich; injured group) intratracheally. Bleomycin-treated animals showing less than $10 \%$ weight loss over the first 14 days were excluded from the study. Injured C57BL/6J animals were treated with ABT-263 (Apex Biotechnology; $50 \mathrm{mg} / \mathrm{kg}$ in 10\% EtOH, 20\% PEG-400, and 70\% Phosal 40) or vehicle control for 7 days starting 1 week after injury. Injured $p 16^{\text {Ink4a }}-3 \mathrm{MR}$ mice received 1 i.p. injection of GCV daily $(25 \mathrm{mg} / \mathrm{kg} / \mathrm{day}$ in PBS; Sigma-Aldrich) for 10 days, starting 10 days after bleomycin injury. Animals were euthanized either 14 or 21 days after bleomycin challenge. To collect BALF, $1 \mathrm{~mL}$ of PBS was injected intratracheally, and approximately $0.8 \mathrm{~mL}$ was retrieved. The BALF was centrifuged at $500 \mathrm{~g}$ for 10 minutes at room temperature. Lungs were collected and distributed as follows: left lung was used for hydroxyproline measurement or was embedded (paraffin or OCT); other lobes were used for RNA/protein extraction.

Assessment of pulmonary fibrosis. The extent of fibrosis indices in lungs was assessed with established markers (55). Collagen deposition was quantified using hydroxyproline measurements and Picrosirius red staining and quantification. Hydroxyproline content was measured as described (55). The left lobe of the lung was homogenized briefly in $1 \mathrm{~mL}$ of water and incubated in 50\% trichloroactic acid for 20 minutes. Samples were then hydrolyzed with $6 \mathrm{~N} \mathrm{HCl}$ at $110^{\circ} \mathrm{C}$ for 24 hours, reconstituted in $2 \mathrm{~mL}$ of water, and agitated at room temperature for 2 hours. Samples were then mixed in equal parts chloramine $\mathrm{T}(1.4 \%$ in $0.5 \mathrm{M}$ sodium acetate and $10 \%$ isopropanol) and Ehrlich's solution (1 M p-dimethylaminobenzaldehyde in 70\% isopropanol and 
$30 \%$ perchloric acid) and heated at $65^{\circ} \mathrm{C}$ for 15 minutes. Absorbance was then measured at $550 \mathrm{~nm}$. Standard curves were generated for each experiment using a hydroxyproline standard (trans-4-hydro-1-proline). Results are expressed as $\mathrm{mg}$ hydroxyproline $/ \mathrm{mL}$ tissue extract. Paraffin sections (5- $\mu \mathrm{m}$ thick) were layered on silane-coated slides and stained with Picrosirius red to quantify parenchymal collagen deposition. The level of staining was assessed with ImagePro software v6.2 (Media Cybernetics Inc.) and expressed as a percentage of the total area of the image analyzed. To minimize variation, samples were processed under identical conditions. Five slides of the entire left lung per animal were quantified by an operator blinded to the treatments.

Isolation of $\mathrm{CD} 45^{+}$by flow cytometry. Mouse lung macrophages were isolated from PBS- or bleomycin-injured lungs 14 days after injection as previously described (56). Single cell preparations were incubated for 1 hour at $4^{\circ} \mathrm{C}$ with APC-Cy7 rat anti-mouse CD45 antibody (1:1:00, BioLegend, 103116) in sort buffer (DMEM without phenol red plus 2\% FBS [Thermo Fisher Scientific]). Cells were resuspended in sort buffer plus Sytox Blue (1:1000; Thermo Fisher Scientific; S34857), and sorting was performed on BD FACS Aria cytometers. CD $45^{+}$were then cultured in DMEM $+10 \%$ FBS for 48 hours in the presence of DMSO (vehicle control) or $10 \mathrm{mM} \mathrm{ABT-263.} \mathrm{RNA} \mathrm{extraction} \mathrm{and} \mathrm{qPCR} \mathrm{were} \mathrm{performed} \mathrm{as} \mathrm{previously} \mathrm{described.}$

\section{Human tissues}

Detection of senescent cells expressing ALOX5, SPC, aSMA, and $16^{\text {Ink4a }}$ in human lung tissue. As previously described (56), $7-\mu \mathrm{m}$ thick lung cryosections were cut and incubated with $0.1 \%$ sodium borohydride (Sigma-Aldrich) in PBS to reduce aldehyde-induced background fluorescence for three 10-minute intervals and were subsequently blocked and stained in PBS plus 1\% BSA (Affymetrix), 5\% nonimmune horse serum, 0.1\% Triton X-100, and $0.02 \%$ sodium azide. Subsequently, tissue sections were stained with rabbit anti-human ALOX5 (catalog ab169788, Abcam), goat anti-human SPC (clone C19, Santa Cruz Biotechnology Inc.), mouse anti-human aSMA (clone 1A4, MilliporeSigma), and mouse anti-human $p 16^{\text {Inkta }}$ (catalog 725-4793, Roche Diagnostics) and. Mosaic pictures were taken randomly and over 2,000 cells (DAPI ${ }^{+}$cells) per section were counted.

Isolation of lung fibroblasts. Distal lung tissue was obtained. Using sterile techniques, tissue was dissected into fragments of approximately $1 \mathrm{~mm}^{2}$. The tissue fragments were then incubated for 96 hours with DMEM $+10 \%$ FBS $+1 \times$ antibiotic/antimycotic (Thermo Fisher Scientific). Fibroblasts crawl out of the tissue fragments and attach to the plate. Pulmonary fibroblasts were then plated into 6-well plates, irradiated as previously described to induce senescence. Ten days after irradiation, total RNA was extracted and mRNA expression of LT and PG biosynthesis enzymes was analyzed by qPCR as previously described.

\section{Statistics}

One-way ANOVA analyses were used for multiple comparisons. Comparisons between groups were performed using the 2-tailed Student's $t$ test or Mann-Whitney $U$ test (for comparison of data sets with nonequivalent variances). Heatmaps use $P<0.05$ for all entities. All data are presented as mean \pm SEM. All analyses were performed in GraphPad Prism 7.0. $P<0.05$ was considered statistically significant.

\section{Study approval}

All human tissue samples and primary fibroblasts from IPF and normal subjects were obtained from UCSF Interstitial Lung Disease Blood and Tissue Repository. They are classified as "nonidentifiable otherwise discarded human tissues." Animal experiments were conducted using a protocol approved by the IACUC of the Buck Institute for Research on Aging and UCSF.

\section{Author contributions}

CDW was responsible for the study concept related to senescence and execution, interpretated culture and LT studies, and cowrote the manuscript. SSD measured LT and PG levels in all samples. JRJ conducted p16 $6^{\text {Ink4a }}-3 \mathrm{MR}$ study and hydroxyproline assay. AV conducted in vivo experiments. CC performed Picrosirius red staining. ANB conducted flow sorting, immunochemistry assays, and macrophage in vitro assays. CAC assisted in the in vitro assays. RR performed in vitro assays with LT antagonists and collected cell count data. YW performed Western blots. HAC was responsible for study design, data interpretation, and cowriting of the manuscript. AR supervised and interpreted data from SSD. CJLS was responsible for study concept related to pulmonary fibrosis, conducted assays related to human samples, interpretated in vivo studies, and cowrote the manuscript. FA maintained the 3MR mouse colony and assisted in experiments. JC designed the study, interpretated data, and cowrote the manuscript. 


\section{Acknowledgments}

This work was supported by NIH grants AG009909 (JC), AG051729 (JC), and AG052744 (CJLS).

Address correspondence to: Claude Jourdan Le Saux, UCSF, 513 Parnassus Avenue, HSE-201 Box 0111, San Francisco, California 94143-0130, USA. Phone: 415.514.1210; Email: claude.lesaux@ucsf.edu.

1. Hutchinson JP, McKeever TM, Fogarty AW, Navaratnam V, Hubbard RB. Increasing global mortality from idiopathic pulmonary fibrosis in the twenty-first century. Ann Am Thorac Soc. 2014;11(8):1176-1185.

2. Selman M, King TE, Pardo A, American Thoracic Society, European Respiratory Society, American College of Chest Physicians. Idiopathic pulmonary fibrosis: prevailing and evolving hypotheses about its pathogenesis and implications for therapy. Ann Intern Med. 2001;134(2):136-151.

3. Gauldie J. Pro: Inflammatory mechanisms are a minor component of the pathogenesis of idiopathic pulmonary fibrosis. Am J Respir Crit Care Med. 2002;165(9):1205-1206.

4. Balestro E, et al. Immune Inflammation and Disease Progression in Idiopathic Pulmonary Fibrosis. PLoS ONE. 2016;11(5):e0154516.

5. Chilosi M, Carloni A, Rossi A, Poletti V. Premature lung aging and cellular senescence in the pathogenesis of idiopathic pulmonary fibrosis and COPD/emphysema. Transl Res. 2013;162(3):156-173.

6. Akram KM, Lomas NJ, Forsyth NR, Spiteri MA. Alveolar epithelial cells in idiopathic pulmonary fibrosis display upregulation of TRAIL, DR4 and DR5 expression with simultaneous preferential over-expression of pro-apoptotic marker p53. Int J Clin Exp Pathol. 2014;7(2):552-564.

7. Minagawa S, et al. Accelerated epithelial cell senescence in IPF and the inhibitory role of SIRT6 in TGF- $\beta$-induced senescence of human bronchial epithelial cells. Am J Physiol Lung Cell Mol Physiol. 2011;300(3):L391-L401.

8. Alder JK, et al. Short telomeres are a risk factor for idiopathic pulmonary fibrosis. Proc Natl Acad Sci USA. 2008;105(35):13051-13056.

9. Campisi J, d'Adda di Fagagna F. Cellular senescence: when bad things happen to good cells. Nat Rev Mol Cell Biol. 2007;8(9):729-740.

10. Demaria M, et al. An essential role for senescent cells in optimal wound healing through secretion of PDGF-AA. Dev Cell. 2014;31(6):722-733.

11. Shivshankar P, Brampton C, Miyasato S, Kasper M, Thannickal VJ, Le Saux CJ. Caveolin-1 deficiency protects from pulmonary fibrosis by modulating epithelial cell senescence in mice. Am J Respir Cell Mol Biol. 2012;47(1):28-36.

12. Calhoun C, et al. Senescent Cells Contribute to the Physiological Remodeling of Aged Lungs. J Gerontol A Biol Sci Med Sci. 2016;71(2):153-160.

13. Lehmann M, et al. Senolytic drugs target alveolar epithelial cell function and attenuate experimental lung fibrosis ex vivo. Eur Respir J. 2017;50(2):1602367.

14. Schafer MJ, et al. Cellular senescence mediates fibrotic pulmonary disease. Nat Commun. 2017;8:14532.

15. Le Saux CJ, et al. A novel telomerase activator suppresses lung damage in a murine model of idiopathic pulmonary fibrosis. PLoS ONE. 2013;8(3):e58423.

16. Coppé JP, et al. Senescence-associated secretory phenotypes reveal cell-nonautonomous functions of oncogenic RAS and the p53 tumor suppressor. PLoS Biol. 2008;6(12):2853-2868.

17. Acosta JC, et al. Chemokine signaling via the CXCR2 receptor reinforces senescence. Cell. 2008;133(6):1006-1018.

18. Zdanov S, et al. Normal or stress-induced fibroblast senescence involves COX-2 activity. Exp Cell Res. 2007;313(14):3046-3056

19. Catalano A, Rodilossi S, Caprari P, Coppola V, Procopio A. 5-Lipoxygenase regulates senescence-like growth arrest by promoting ROS-dependent p53 activation. EMBO J. 2005;24(1):170-179.

20. Maher TM, et al. Diminished prostaglandin E2 contributes to the apoptosis paradox in idiopathic pulmonary fibrosis. $A m J$ Respir Crit Care Med. 2010;182(1):73-82.

21. Dackor RT, et al. Prostaglandin E(2) protects murine lungs from bleomycin-induced pulmonary fibrosis and lung dysfunction. Am J Physiol Lung Cell Mol Physiol. 2011;301(5):L645-L655.

22. Hodges RJ, et al. Severity of lung injury in cyclooxygenase-2-deficient mice is dependent on reduced prostaglandin E(2) production. Am J Pathol. 2004;165(5):1663-1676.

23. Kowal-Bielecka O, et al. Cyclooxygenase- and lipoxygenase-derived eicosanoids in bronchoalveolar lavage fluid from patients with scleroderma lung disease: an imbalance between proinflammatory and antiinflammatory lipid mediators. Arthritis Rheum. 2005;52(12):3783-3791.

24. Wilborn J, Crofford LJ, Burdick MD, Kunkel SL, Strieter RM, Peters-Golden M. Cultured lung fibroblasts isolated from patients with idiopathic pulmonary fibrosis have a diminished capacity to synthesize prostaglandin E2 and to express cyclooxygenase-2. J Clin Invest. 1995;95(4):1861-1868

25. Kalish BT, Kieran MW, Puder M, Panigrahy D. The growing role of eicosanoids in tissue regeneration, repair, and wound healing. Prostaglandins Other Lipid Mediat. 2013;104-105:130-138.

26. Haeggström JZ, Funk CD. Lipoxygenase and leukotriene pathways: biochemistry, biology, and roles in disease. Chem Rev. 2011;111(10):5866-5898.

27. Castelino FV. Lipids and eicosanoids in fibrosis: emerging targets for therapy. Curr Opin Rheumatol. 2012;24(6):649-655.

28. Lin LL, Wartmann M, Lin AY, Knopf JL, Seth A, Davis RJ. cPLA2 is phosphorylated and activated by MAP kinase. Cell. 1993;72(2):269-278.

29. Kramer RM, et al. p38 mitogen-activated protein kinase phosphorylates cytosolic phospholipase A2 (cPLA2) in thrombin-stimulated platelets. Evidence that proline-directed phosphorylation is not required for mobilization of arachidonic acid by cPLA2. J Biol Chem. 1996;271(44):27723-27729.

30. Freund A, Patil CK, Campisi J. p38MAPK is a novel DNA damage response-independent regulator of the senescence-associat- 
ed secretory phenotype. EMBO J. 2011;30(8):1536-1548.

31. Wiley CD, et al. Mitochondrial Dysfunction Induces Senescence with a Distinct Secretory Phenotype. Cell Metab. 2016;23(2):303-314

32. Garrison G, et al. Reversal of myofibroblast differentiation by prostaglandin E(2). Am J Respir Cell Mol Biol. 2013;48(5):550-558

33. Penke LR, Huang SK, White ES, Peters-Golden M. Prostaglandin E2 inhibits $\alpha$-smooth muscle actin transcription during myofibroblast differentiation via distinct mechanisms of modulation of serum response factor and myocardin-related transcription factor-A. J Biol Chem. 2014;289(24):17151-17162.

34. Izumo T, Kondo M, Nagai A. Cysteinyl-leukotriene 1 receptor antagonist attenuates bleomycin-induced pulmonary fibrosis in mice. Life Sci. 2007;80(20):1882-1886.

35. Peters-Golden M, et al. Protection from pulmonary fibrosis in leukotriene-deficient mice. Am J Respir Crit Care Med. 2002;165(2):229-235

36. Beller TC, Friend DS, Maekawa A, Lam BK, Austen KF, Kanaoka Y. Cysteinyl leukotriene 1 receptor controls the severity of chronic pulmonary inflammation and fibrosis. Proc Natl Acad Sci USA. 2004;101(9):3047-3052.

37. Oga T, et al. Prostaglandin F(2alpha) receptor signaling facilitates bleomycin-induced pulmonary fibrosis independently of transforming growth factor-beta. Nat Med. 2009;15(12):1426-1430.

38. Chang J, et al. Clearance of senescent cells by ABT263 rejuvenates aged hematopoietic stem cells in mice. Nat Med. 2016;22(1):78-83

39. Lagares D, et al. Targeted apoptosis of myofibroblasts with the BH3 mimetic ABT-263 reverses established fibrosis. Sci Transl Med. 2017;9(420):eaal3765.

40. Moeller A, Ask K, Warburton D, Gauldie J, Kolb M. The bleomycin animal model: a useful tool to investigate treatment options for idiopathic pulmonary fibrosis? Int J Biochem Cell Biol. 2008;40(3):362-382.

41. Izbicki G, Segel MJ, Christensen TG, Conner MW, Breuer R. Time course of bleomycin-induced lung fibrosis. Int J Exp Pathol. 2002;83(3):111-119.

42. Bauman KA, et al. The antifibrotic effects of plasminogen activation occur via prostaglandin E2 synthesis in humans and mice. J Clin Invest. 2010;120(6):1950-1960.

43. Liu X, Sun SQ, Ostrom RS. Fibrotic lung fibroblasts show blunted inhibition by cAMP due to deficient cAMP response element-binding protein phosphorylation. J Pharmacol Exp Ther. 2005;315(2):678-687.

44. Waters DW, et al. Fibroblast senescence in the pathology of idiopathic pulmonary fibrosis. Am J Physiol Lung Cell Mol Physiol. 2018;315(2):L162-L172.

45. Coppé JP, Desprez PY, Krtolica A, Campisi J. The senescence-associated secretory phenotype: the dark side of tumor suppression. Annu Rev Pathol. 2010;5:99-118.

46. Hernandez-Segura A, de Jong TV, Melov S, Guryev V, Campisi J, Demaria M. Unmasking Transcriptional Heterogeneity in Senescent Cells. Curr Biol. 2017;27(17):2652-2660.e4.

47. Pan J, et al. Inhibition of Bcl-2/xl With ABT-263 Selectively Kills Senescent Type II Pneumocytes and Reverses Persistent Pulmonary Fibrosis Induced by Ionizing Radiation in Mice. Int J Radiat Oncol Biol Phys. 2017;99(2):353-361.

48. Krizhanovsky V, et al. Senescence of activated stellate cells limits liver fibrosis. Cell. 2008;134(4):657-667.

49. Tsuji T, Aoshiba K, Nagai A. Alveolar cell senescence in patients with pulmonary emphysema. Am J Respir Crit Care Med. 2006;174(8):886-893.

50. Wilborn J, Bailie M, Coffey M, Burdick M, Strieter R, Peters-Golden M. Constitutive activation of 5-lipoxygenase in the lungs of patients with idiopathic pulmonary fibrosis. J Clin Invest. 1996;97(8):1827-1836.

51. Petkova DK, Clelland CA, Ronan JE, Lewis S, Knox AJ. Reduced expression of cyclooxygenase (COX) in idiopathic pulmonary fibrosis and sarcoidosis. Histopathology. 2003;43(4):381-386.

52. Borok Z, et al. Augmentation of functional prostaglandin E levels on the respiratory epithelial surface by aerosol administration of prostaglandin E. Am Rev Respir Dis. 1991;144(5):1080-1084.

53. Phan SH, McGarry BM, Loeffler KM, Kunkel SL. Leukotriene C4 binds to rat lung fibroblasts and stimulates collagen synthesis. Adv Prostaglandin Thromboxane Leukot Res. 1987;17B:997-999.

54. Wiley CD, Davis S, Ramanathan A. Measurement of Metabolite Changes in Senescent Cells by Mass Spectrometry. Methods Mol Biol. 2019;1896:139-147.

55. Huang WT, et al. Plasminogen activator inhibitor 1, fibroblast apoptosis resistance, and aging-related susceptibility to lung fibrosis. Exp Gerontol. 2015;61:62-75.

56. Chapman HA, et al. Integrin $\alpha 6 \beta 4$ identifies an adult distal lung epithelial population with regenerative potential in mice. $J$ Clin Invest. 2011;121(7):2855-2862. 